\title{
A Frequency-based Parent Selection for Reducing the Effect of Evaluation Time Bias in Asynchronous Parallel Multi-objective Evolutionary Algorithms
}

\author{
Tomohiro Harada
}

Received: date / Accepted: date

\begin{abstract}
This paper proposes a new parent selection method for reducing the effect of evaluation time bias in asynchronous parallel evolutionary algorithms (APEAs). APEAs have the advantage of increasing the computational efficiency even when the evaluation times of solutions differ. However, APEAs have a problem that their search direction is biased toward the search region with a short evaluation time. The proposed parent selection method considers the search frequency of solutions to reduce such an adverse influence of APEAs while maintaining their computational efficiency. We conduct experiments on toy problems that reproduce the evaluation time bias on multi-objective optimization problems to investigate the effectiveness of the proposed method. The experiments use NSGA-III, a well-known multiobjective evolutionary algorithm. In the experiments, we compare the proposed method with the synchronous and the asynchronous methods. The experimental results reveal that the proposed method can reduce the effect of the evaluation time bias while reducing the computing time of the parallel NSGA-III.
\end{abstract}

Keywords Evolutionary algorithm · parallelism · asynchronous evaluation · evaluation time bias · parent selection $\cdot$ multi-objective optimization

\section{Introduction}

Evolutionary algorithms (EAs) have been applied to a wide range of real-world optimization problems such as engineering [23], pathfinding [25], data mining [29],

\section{T. Harada}

6-6 Asahigaoka, Hino, Tokyo, Japan

Faculty of System Design, Tokyo Metropolitan University

E-mail: harada@tmu.ac.jp nanoscience [28], power system [32], and so on because of their high search capability without any problemspecific knowledge. When applying EAs to real-world optimization problems, solution evaluations may take much computational time due to physical simulation or actual consumption time measurement.

Since a parallelization of fitness evaluations is a viable option to speed up the optimization process in such a situation, many researchers have studied parallel EAs (PEAs) $[2,3,12]$. A master-slave parallelization [9] is one of the most potent approaches of PEAs. On a master-slave PEA, a single master computational node executes the main procedure of an EA, e.g., initialization, parent selection, genetic operations, replacement. In contrast, many slave nodes evaluate each solution in parallel.

A master-slave PEA can be mainly classified into two approaches, a synchronous PEA (SPEA) and an asynchronous PEA (APEA). An SPEA generates a population in the next generation after evaluating all solutions. On the other hand, an APEA generates a new solution immediately after completing one solution evaluation. Since an SPEA generates new solutions after slave nodes evaluate all solutions, it needs to wait for a solution with the longest evaluation time when the evaluation times are excessively different. This causes waste of the idling time of slave nodes, and the computational efficiency decreases. Besides this, since an APEA generates a new solution without waiting for other solution evaluations, it can continuously evolve solutions without the idling time of slave nodes. However, since an APEA generates a new solution whenever a solution evaluation completes, it is possible to fall into local optima with a short evaluation time $[10,26,27]$.

This paper proposes a new parent selection method to reduce the effect of evaluation time bias in APEAs. 
One primordial matter of APEAs being affected by the evaluation time bias is that many opportunities of parent selections are given to solutions with a short evaluation time. This induces an excessive search to the region with a short evaluation time, even if it is a local optimum. Thus, the proposed method considers the search progress of each search region and selects parents so that the search frequency of all solutions becomes uniform. The proposed method introduces a new parameter to retain the search frequency of solutions and selects parents according to this frequency parameter.

In order to investigate the effectiveness of the proposed method, this paper conducts experiments using multi-objective optimization problems. This paper designs toy problems having the evaluation time bias by using multimodal multi-objective test function (MMF) [33]. MMFs are bi-objective optimization problems with separate Pareto sets in different regions of the decision variable space. Our toy problems have two Pareto sets with long and short evaluation times. In such problems, it is expected that APEAs precisely obtain solutions in the region with a short evaluation time, while the proposed method acquires both Pareto sets equally. This paper uses the computational model proposed in [36] to simulate a parallel computing environment and employs NSGA-III [7] as the multi-objective evolutionary algorithm (MOEA). The experiments compare synchronous parallel NSGA-III, asynchronous parallel NSGA-III, and asynchronous parallel NSGA-III using the proposed selection method.

This paper is an extended version of the work of [11] presented by the author. The essential difference from the previous work is as follows:

- Although the previous work tested the proposed method on the DTLZ1 problem with a single Pareto set, this paper utilizes the MMF problems with separate Pareto sets to deeply and clearly analyze the effect of the evaluation time bias.

- This paper provides further analysis of a parameter of the proposed method, in particular, $r_{s}$ in the proposed method (see Section 3.2), which was fixed in the previous work.

The rest of this paper is organized as follows. The following section briefly introduces APEAs and mentions their problems in the evaluation time bias. Section 3 proposes the parent selection strategy and shows its concrete example on NSGA-III. Section 4 defines the toy problems used in this work, and Section 5 describes the experimental settings. Then, Section 6 shows and analyzes the experimental results by comparing the proposed method with the synchronous and asynchronous methods, and the parameter setting of the proposed

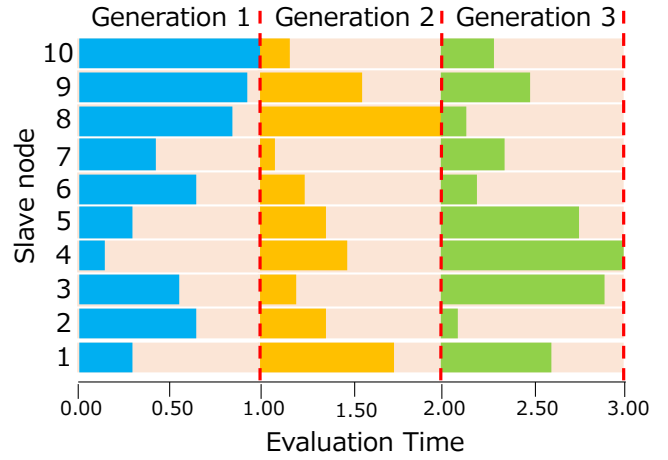

(a) SPEA

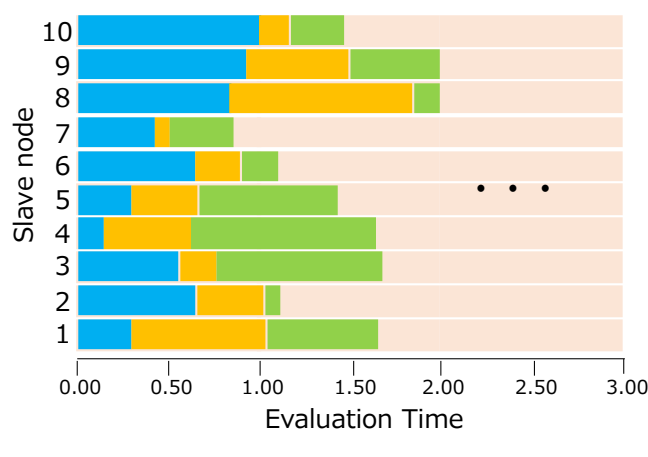

(b) APEA

Fig. 1: Illustrations of SPEA and APEA on the parallel computing environment with ten slave nodes

method is discussed in Section 7. Finally, Section 8 concludes this paper and addresses future works.

\section{Background}

Parallel evolutionary algorithms (PEAs) have been widely studied to reduce the computing time of EA methods by executing a single EA run on multiple computing nodes $[3,2,12]$. Although several PEA approaches were proposed, a master-slave parallelization is a straightforward approach to implement PEAs $[9,20]$. A masterslave PEA executes the main procedure of EAs, i.e., initialization, selection, genetic operators, and replacement, on one master computing node. On the other hand, many slave nodes evaluate newly generated solutions in parallel and return their results to the master node.

The master-slave PEAs can be classified into synchronous PEAs (SPEAs) and asynchronous PEAs (APEAs). Fig. 1 illustrates an SPEA and an APEA. An SPEA, shown in Fig. 1a, waits for all evaluations of solutions executed by slave nodes and generates a new population using all newly evaluated solutions. Since an SPEA needs to wait for all evaluation solutions for each gener- 


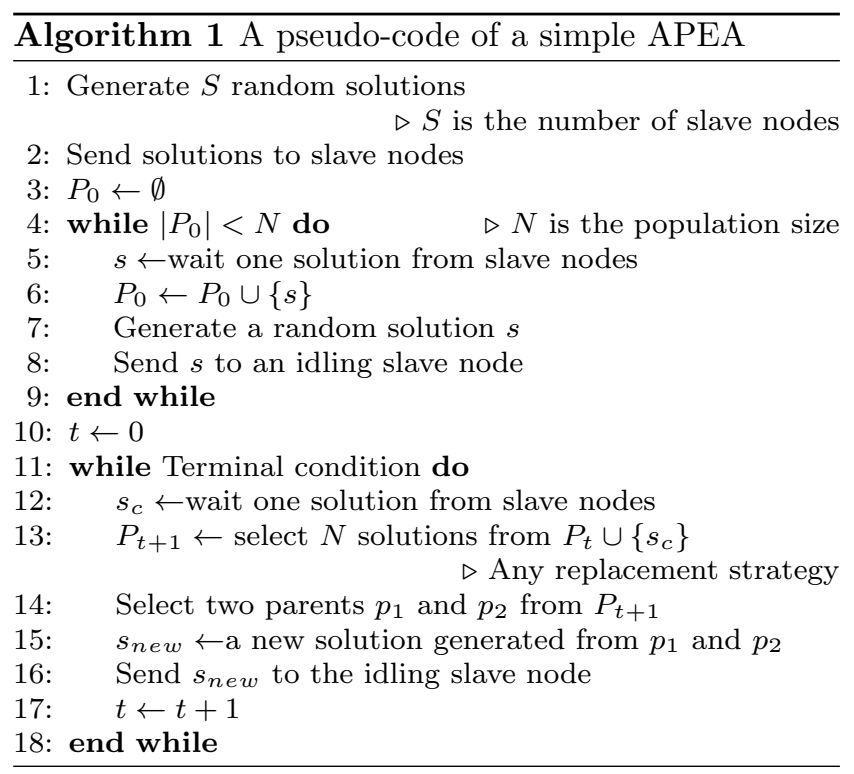

ation, it wastes much idling time if the evaluation times differ, decreasing computational efficiency. On the other hand, an APEA, shown in Fig. 1b, continuously generates a new solution without waiting for evaluations of other solutions. This enables the efficient use of the computing resource even if the evaluation times of solutions differ.

A pseudo-code of a simple APEA is shown in Algorithm 1. The initial population of $P_{0}$ is generated randomly or using a problem specific way until $P_{0}$ is filled with $N$ solutions ( $N$ is the population size). After that, a solution evaluation is waited for from slave nodes. When one solution evaluation $s_{c}$ completes, the population $P_{t}$ is replaced with any replacement method. Then, a new solution $s_{n e w}$ is then generated and sent to the idling slave node to be evaluated. This loop is continued until a terminal condition is satisfied.

Several previous research have demonstrated the effectiveness of APEAs, for example, on program optimizations [13,14], continuous optimizations [17,34], and multi-objective optimizations $[8,30]$. However, the previous studies demonstrated that an APEA is biased toward the search region having a short evaluation time if the evaluation time differs depends on the search region $[10,27,26]$. This is because an APEA gives many opportunities of searching solutions with a short evaluation time since it generates a new solution immediately after one solution evaluation completes.

On the other hand, an SPEA is not affected by the evaluation time bias because it can evenly search solutions independent of their evaluation times. However, an SPEA still wastes waiting time if the evaluation time is biased, so it needs much computing time for the optimization. The work of [24] proposed an inter- leaving generation evolutionary algorithm (IGEA) that improves the computing efficiency of an SPEA by reducing the waste of the waiting time of the slave nodes. In particular, IGEA generates offspring when their parents complete their evaluations and starts their evaluations on the slave nodes. This is possible because, in general, a few parent solutions are needed to generate an offspring individual, and it is enough to wait for the evaluations of parent solutions. The previous study found that IGEA can improve the computing efficiency of $(\lambda, \lambda)$-EA and $(\lambda+\lambda)$-EA on several variations of the evaluation time bias compared with a simple SPEA. Subsequent studies have proposed its improved methods [22] and the application of the interleaving generation mechanism to differential evolution (DE) [21]. However, the computing efficiency of IGEA and its variants decreases as increasing the number of slave nodes. In addition, the applicable range of IGEA is limited and can be applied to only EAs satisfying some restrictions.

\section{Proposed method}

This section proposes a new parent selection strategy for reducing the effect of evaluation time bias in APEAs. The following subsection explains the basic concept of the proposed method, and then Section 3.2 introduces the proposed parent selection. Finally, Section 3.3 shows an example of applying the proposed method to the asynchronous parallel NSGA-III.

\subsection{Basic concept}

APEAs are adversely affected by the evaluation time bias because the search frequency to solutions with a short evaluation time increases. On the other hand, SPEAs are not affected by the evaluation time bias because the search frequency is almost the same in all search regions regardless of the evaluation time bias. This fact suggests that it is helpful to adjust the search progress of all solutions to be uniform for reducing the effect of evaluation time bias in APEAs.

Therefore, this paper proposes a new parent selection method that introduces a new parameter of solutions. In particular, this parameter retains the number of ancestors of each solution (i.e., how many times the genetic operations are performed to generate each solution) as the search frequency. The proposed selection method considers this parameter and selects parents to preserve the uniformity of the search frequency of solutions. This is expected to prevent excessive parent selection of solutions in the regions with short evaluation 


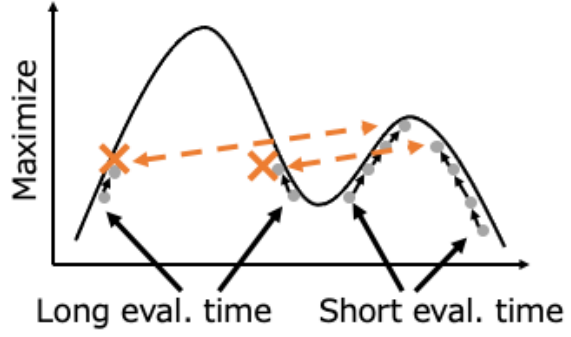

(a) Conventional APEAs

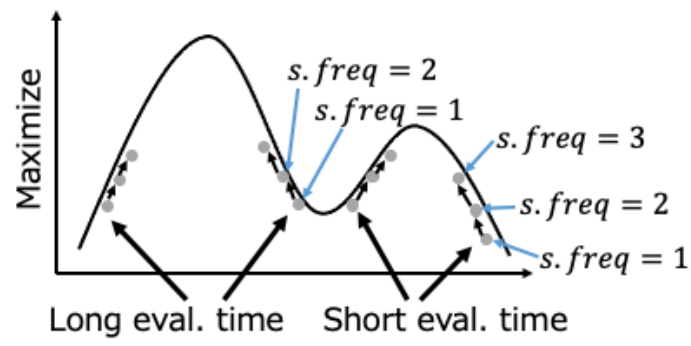

(b) The proposed parent selection

Fig. 2: Illustrations of the conventional APEAs and the proposed method

times and avoid the search bias for the search regions with short evaluation times.

Fig. 2 illustrates the issue of the conventional APEAs and the concept of the proposed method. In these figures, the horizontal axis shows the one-dimensional search space, while the vertical axis shows the maximization objective function. This example has a global optimum with a long evaluation time and a local optimum with a short evaluation time. The conventional APEAs shown in Fig. 2a frequently search solutions in the region with a short evaluation time (the right region in this figure) so that solutions close to the global optimum (a long evaluation time) are eliminated by comparing them with the more frequently searched solutions. To overcome this problem, the proposed method shown in Fig. $2 \mathrm{~b}$ retains the search frequency of solutions (s.freq in the figure) and attempts to preserve the uniformity of these frequencies. This may avoid eliminating solutions with a long evaluation time due to its slow search progress.

\subsection{Algorithm}

For establishing the parent selection method in consideration of the search frequency of solutions, this paper introduces a new parameter to retain the search frequency of each solution. Let the search frequency of a solution $s$ be $s$.freq. In the initial population, the search frequency parameter for all solutions s.freq $\overline{\text { Algorithm } 2 \text { A pseudo-code of a simple APEA with }}$ the proposed parent selection method. The underlined texts are specific to the proposed method.

1: Generate $S$ random solutions $\triangleright S$ is the number of slave nodes

2: s.freq $\leftarrow 1$ for all generated solutions

3: Send solutions to slave nodes

4: $P_{0} \leftarrow \emptyset$

5: while $\left|P_{0}\right|<N$ do $\quad \triangleright N$ is the population size

6: $\quad s \leftarrow$ wait one solution from slave nodes

7: $\quad P_{0} \leftarrow P_{0} \cup\{s\}$

8: $\quad$ Generate a random solution $s$

9: $\quad$ s.freq $\leftarrow 1$

10: $\quad$ Send $s$ to an idling slave node

11: end while

12: $t \leftarrow 0$

13: while Terminal condition do

14: $\quad s_{c} \leftarrow$ wait one solution from slave nodes

15: $\quad P_{t+1} \leftarrow$ select $N$ solutions from $P_{t} \cup\left\{s_{c}\right\}$ $\triangleright$ Any replacement strategy

16: $\quad$ Sort $P_{t+1}$ in ascending order of s.freq $\left(s \in P_{t+1}\right)$

17: $\quad \overline{P_{t+1}^{\prime} \leftarrow \text { the first } r_{s}\left|P_{t+1}\right| \text { solutions of } P_{t+1}}$

18: $\quad$ Select two parents $p_{1}$ and $p_{2}$ from $P_{t+1}^{\prime}$

19: $\quad p_{i}$. freq $\leftarrow p_{i}$. freq $+1(i=\{1,2\})$

20: $\quad \frac{p_{i}}{s_{\text {new }} \leftarrow \text { a new solution generated from } p_{1} \text { and } p_{2}}$

21: $\quad s_{\text {new }}$. freq $\leftarrow\left(p_{1}\right.$.freq $+p_{2}$. freq $) / 2$

22: $\quad$ Send $s_{\text {new }}$ to the idling slave node

23: $\quad t \leftarrow t+1$

24: end while

is initialized to 1. After that, whenever a solution is selected as a parent, the search frequency parameter is incremented as $s . f r e q \leftarrow s . f r e q+1$. In addition, generated offspring $s_{\text {new }}$ inherits the search frequency parameters of its parent solutions. In particular, the search frequency parameter of generated offspring is set as the mean of the search frequency parameters of the parent individual. For example, when an offspring solution $s_{n e w}$ is generated from two parents $p_{1}$ and $p_{2}$, the search frequency parameter of $s_{\text {new }}$ is calculated as $s_{n e w} . f r e q \leftarrow\left(p_{1}\right.$. freq $+p_{2}$. freq $) / 2$. This allows us to retain the search progress of each search region as additional information for each solution.

The proposed method pre-selects candidate solutions for the parent selection depending on the search frequency parameter. Although the conventional parent selection selects solutions from the entire population, the proposed method selects parents from only solutions in the population with small search frequency parameters. In other words, the proposed selection method only focuses on solutions that are less searched during the evolution so far. This can reduce the number of search opportunities for solutions with short evaluation times and encourages the entire population to be searched uniformly.

Algorithm 2 shows a pseudo-code of an APEA with the proposed parent selection. Newly added procedures 
from Algorithm 1 are underlined. At Steps 2 and 9, the search frequency parameters of the initial solutions are set to 1 . When selecting parent solutions, the frequency parameter is considered. At Step 16, the population is sorted in ascending order of the frequency parameters. Then, the first $r_{s}\left|P_{t+1}\right|$ solutions of $P_{t+1}$ are extracted as a parent candidate set $P_{t+1}^{\prime}$ at Step 17, and parents are selected from $P_{t+1}^{\prime}$. Here, $r_{s}$ is a selection ratio that is a parameter to determine the importance of the search frequency parameter, and its effect will be discussed in Section 7. By this selection, solutions that are frequently searched are excluded from the parent selection. After selecting parents, their frequency parameters are incremented by one at Step 19. The frequency parameter of a newly generated solution inherits that of parent solutions at Step 21.

The difference between standard APEAs and APEAs with the proposed method is that: the standard version selects parents from the entire population regardless of the search frequency. This induces that solutions having short evaluation time frequently get opportunities to be selected as parents, and the search direction is biased toward the search region where evaluation time is short. On the other hand, the proposed method considers the search frequency of solutions and selects parents from less selected solutions for the offspring generation. This mechanism can give opportunities to be selected as parents for all solutions and prevent the asynchronous evolution from being affected by the evaluation time bias.

\subsection{An example of NSGA-III}

This section shows a concrete example of applying the proposed method to the asynchronous parallel NSGAIII, which will be used in our experiments in Sections 6 and 7. NSGA-III [7] is one of the state-of-the-art MOEA methods, which combines the idea of dominance and decomposition.

The brief flow of the master node in the parallel asynchronous NSGA-III is described in Algorithm 3. Many slave nodes evaluate a solution given from a master node and return its evaluation to a master node in this flow. The master node firstly initializes the population and sends all solutions to slave nodes at Steps 2 and 3. After the initialization is completed, the main procedure is repeated until satisfying the termination condition. In the main loop, NSGA-III randomly selects two parent solutions from the current population (RandomSelection in Algorithm 3) and generates offspring through genetic operators such as crossover and mutation (GeneticOperators in Algorithm 3) at Steps 6 and 7 . Then, the master node sends the generated
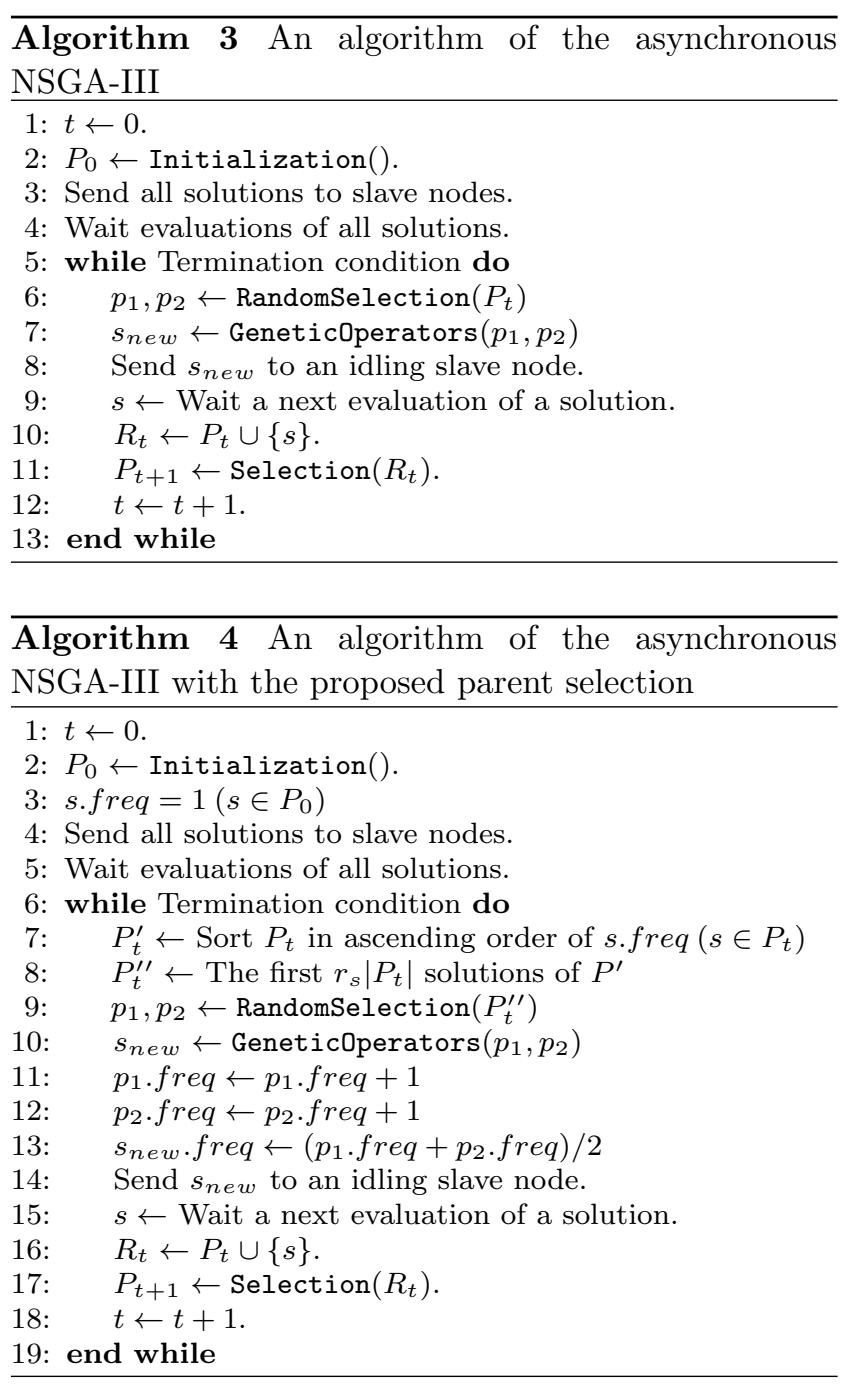

offspring to an idling slave node and waits for the subsequent evaluation of a solution at Steps 8 and 9. When receiving a solution evaluation, NSGA-III selects the next population from the current population and a newly evaluated solution (Selection in Algorithm 3) at Steps 10 and 11. In the selection procedure, NSGA-III uses the front-ranking and the niche-preservation operation based on the reference point.

Algorithm 4 shows an algorithm of the asynchronous parallel NSGA-III with the proposed method. The proposed method first assigns the search frequency parameter $s$. freq $=1$ for all solutions in the initial population $P_{0}$ at Step 3. When generating offspring, the proposed method sorts the population in ascending order of the search frequency parameter and uses the first $r_{s}\left|P_{t}\right|$ solutions for the parent selection at Steps 7-9. After generating offspring, the frequency parameters of the parents are incremented as $p_{i}$. freq $\leftarrow p_{i}$. freq +1 at Steps 11 and 12. In addition, the proposed method updates the frequency parameter of the generated offspring as 
the mean frequencies of its parents as $s_{\text {new }}$. freq $\leftarrow$ $\left(p_{1}\right.$. freq $+p_{2}$. freq $) / 2$ at Step 13 .

\section{Toy problem}

This work designs multi-objective optimization problems with the evaluation time bias to clearly analyze the behavior of the proposed method. In particular, we use the multimodal multi-objective test functions (MMFs) [33], which are bi-objective optimization problems with multiple separate Pareto sets (PS) in different regions of the decision variable space. This study uses MMF2-6 and MMF8 with two-dimensional decision variables, which have two separated PS. The PS for each problem is depicted as Fig. 3 (see Appendix for more detailed problem definitions). Note that this work does not use MMF1 and MMF7 because they have a continuous, non-separate PS. In this work, we define PS with a smaller $x_{2}$ value for each $x_{1}$ value as PS1 while defining another PS as PS2.

Based on the distribution of PS in MMFs, we design the evaluation time in the experiments such that the optimal solutions in PS1 requires a shorter evaluation time while PS2 requires a longer evaluation time. Specifically, we define the biased evaluation time based on the Gaussian function as follows:

$$
\begin{aligned}
t_{\text {bias }}(\boldsymbol{x}) & =t_{\text {mean }}\left(1-\exp \left(-\frac{\left(x_{2}-c_{s}\left(x_{1}\right)\right)^{2}}{2 \sigma^{2}}\right)\right. \\
& \left.+\exp \left(-\frac{\left(x_{2}-c_{l}\left(x_{1}\right)\right)^{2}}{2 \sigma^{2}}\right)\right)
\end{aligned}
$$

where $x_{1}$ and $x_{2}$ are the first and second design variables of $\boldsymbol{x}$. Functions $c_{s}$ and $c_{l}$ are problem-specific functions to determine the center position of the Gaussian function corresponds to the decision variable $x_{1}$. In this work, we name the evaluation time function $t_{\text {bias }}$ Bias. For each problem, $c_{s}$ and $c_{l}$ are the functions defined depending on the shape of PS, while $\sigma$ determines the variance of the Gaussian function. These settings are shown in Table 1.

An example of the evaluation time distribution of MMF2 is shown in Fig. 4. The horizontal axis represents $x_{1}$, while the vertical axis represents $x_{2}$. The color bar indicates the evaluation time. In this setting, solutions in the PS1 in the region where $x_{2} \leq 1$ have a shorter evaluation time than ones in the PS2. In such a situation, it is expected that APEA converges more quickly to the PS1.

In addition to the Bias evaluation time function, this experiment uses a non-biased evaluation time function that returns a random value from the normal distribution as $t_{n o r m}(\boldsymbol{x}) \sim N\left(t_{p}, c_{v} t_{p}\right)$. The variable $t_{p}$
Table 1: The definitions of functions $c_{s}$ and $c_{l}$, and a parameter $\sigma$

\begin{aligned} \hline Problem $\quad &$ Function \\ \hline MMF2 $\quad c_{s}(x) & =\sqrt{x} \\ c_{l}(x) & =1+\sqrt{x} \\ \sigma & =0.25 \\ c_{s}(x) & =\sqrt{x} \\ c_{l}(x) & =0.5+\sqrt{x} \\$ MMF3 & $=0.175 \\$ MMF4 $\quad c_{s}(x) & =\sin (\pi|x|) \\ \quad \sigma & =1+\sin (\pi|x|) \\ c_{s}(x) & =\sin (6 \pi|x-2|+\pi) \\ c_{l}(x) & =\sin (6 \pi|x-2|+\pi)+2 \\ \sigma & =0.25 \\$ MMF5 & \\ $c_{s}(x) & =\sin (6 \pi|x-2|+\pi) \\ c_{l}(x) & =\sin (6 \pi|x-2|+\pi)+1 \\ \sigma & =0.375 \\$ MMF8 $\quad c_{l}(x) & =\sin (|x|)+|x|+4 \\ \sigma & =1.125 \\$ MMF6 & \end{aligned}

denotes the mean evaluation time, while $c_{v}$ determines the variance of the evaluation time. Since $t_{n o r m}(\boldsymbol{x})$ is independent of the decision variable, there is no bias of the evaluation time - this paper names this evaluation time function No-bias.

\section{Experimental setting}

This paper conducts experiments on the simulated parallel computational environment to investigate the effectiveness of the proposed method. We apply the proposed method to NSGA-III as a concrete algorithm shown in Section 3.3. In the experiments, we compare the synchronous parallel NSGA-III, the asynchronous parallel NSGA-III, and the asynchronous parallel NSGAIII with the proposed parent selection. Note that since this paper does not intend to solve multimodal multiobjective optimization problems but use them to simulate the evaluation time bias. Thus, we use a standard NSGA-III, not with some specific technique for finding niches.

This section first explains the simulation environment used in the experiment, and then, we show the 


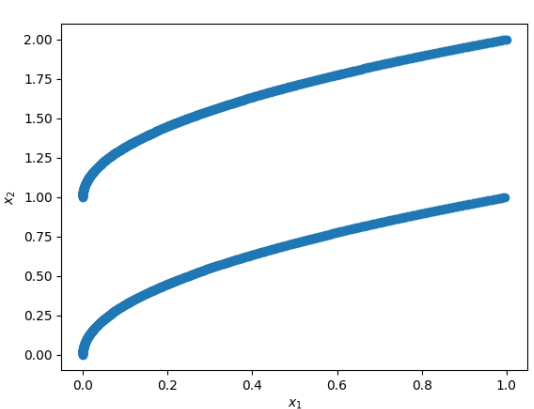

(a) MMF2

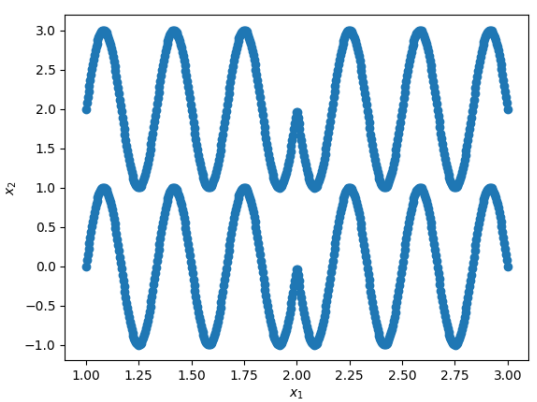

(d) MMF5

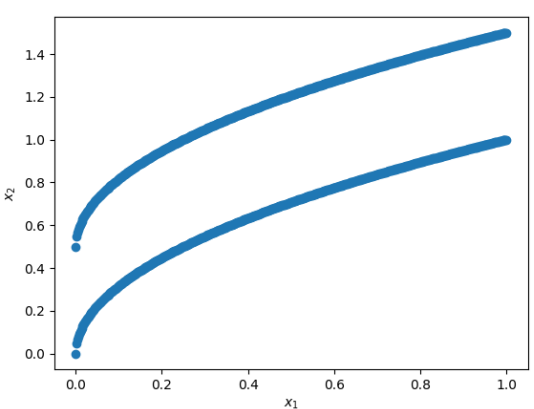

(b) MMF3

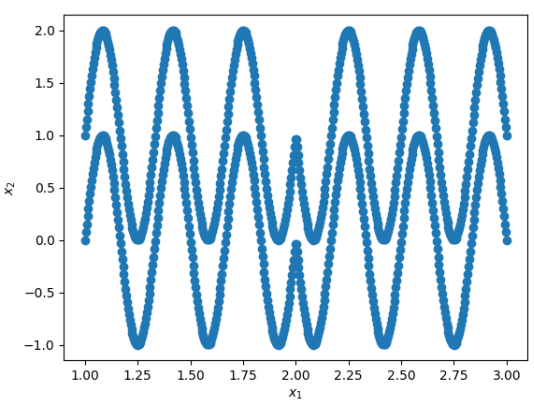

(e) MMF6

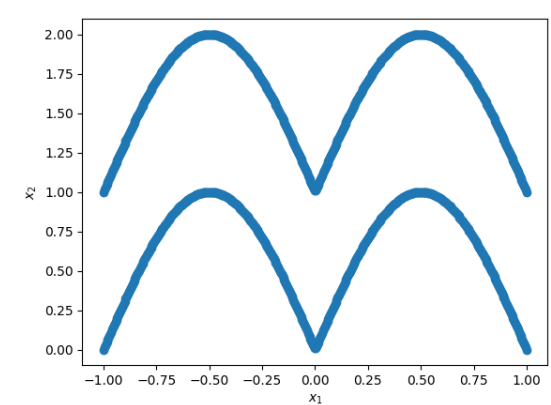

(c) MMF4

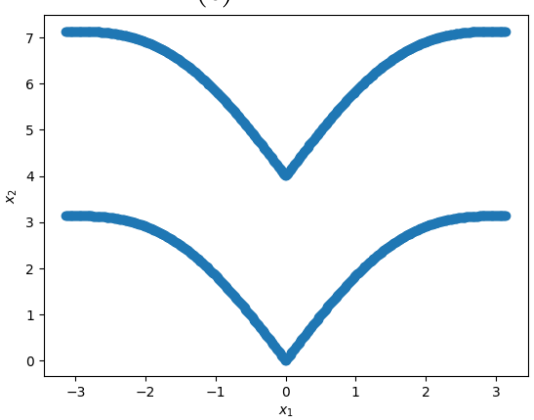

(f) MMF8

Fig. 3: The distribution of Pareto set (PS) in MMFs used in this work

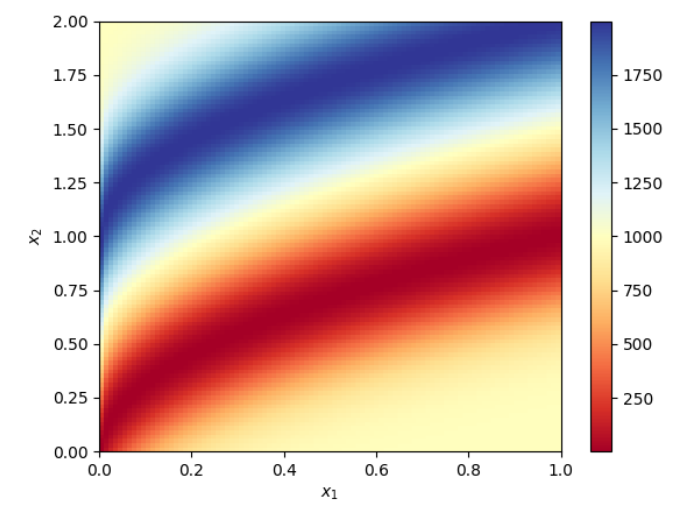

Fig. 4: A biased evaluation time in MMF2 calculated by Eq. (1) where PS1 has a shorter evaluation time than PS2

parameter settings used in the experiment. Finally, we provide evaluation criteria for assessing the competitive methods.

\subsection{Simulated parallel computational environment}

Our experiments use a simulated parallel computational environment based on the computational time model proposed in the work of [36]. This model consists of a single master node and $\lambda$ slave nodes. The master node
Table 2: Parameters

\begin{tabular}{lr}
\hline Parameter & Value \\
\hline Population size & 100 \\
Max. number of evaluations & $8.0 \times 10^{4}$ \\
$p_{c}(\mathrm{SBX})$ & 1.0 \\
$\eta_{c}(\mathrm{SBX})$ & 30.0 \\
$p_{m}(\mathrm{PM})$ & $1 / D$ \\
$\eta_{m}(\mathrm{PM})$ & 20.0 \\
\hline
\end{tabular}

computes the main procedure of the EA algorithm in $t_{s}=1$ simulation time. In contrast, the slave nodes evaluate one solution and return their evaluation results. This experiment simulates $\lambda=100$ slave nodes where 100 solutions are simultaneously evaluated. The evaluation times on the slave nodes depend on the function of Bias and No-bias. In Bias, the value of $t_{\text {mean }}$ is set to 1000 , where the maximum evaluation time is almost 2000, while the minimum one is almost 1 so that the longest evaluation time is 2000 times longer than the shortest one. On the other hand, in No-bias, the mean evaluation time $t_{p}=1000$, while the variance parameter $c_{v}=0.2$.

\subsection{Parameters}

We conduct the experiments for 31 independent runs for each parallelization method. The parameters used in the experiments are shown in Table 2, which are 
commonly used in all NSGA-III variants. The population size is 100, which means all solutions in the population can be evaluated simultaneously in the synchronous NSGA-III. The maximum number of evaluations is $8.0 \times 10^{4}$, which corresponds to 800 generations in the synchronous NSGA-III. As the genetic operator, the simulated binary crossover (SBX) [5] with the probability of $p_{c}=1.0$ and the distribution index of $\eta_{c}=30.0$ is used, and the polynomial mutation $(\mathrm{PM})[6]$ with the probability of $p_{m}=1 / D$ and the distribution index of $\eta_{m}=20.0$.

\subsection{Evaluation criteria}

This experiment uses the $I G D$ indicator [4] to assess the quality of the obtained solutions in the objective space. At the same time, the $I G D X$ indicator [35] is also used for evaluating the quality of solutions in the design variable space. The $I G D$ indicator is calculated as:

$I G D\left(P^{*}, P\right)=\sum_{p \in P^{*}} \frac{d(p, P)}{\left|P^{*}\right|}$

where $P^{*}$ denotes a set of reference points (the true Pareto front in this work) in the calculation of $I G D$, while $P$ denotes the non-dominated solutions generated by the algorithm. $d(p, P)$ calculates the nearest Euclidean distance from $p$ to solutions in $P$. The solutions obtained by the algorithm are worthful if the $I G D$ value is small. When calculating $I G D$ on the objective space, the Euclidean distance is calculated on the objective space (i.e., $p$ in Eq. (8) is the objective value vector). On the other hand, when calculating $I G D X$, the Euclidean distance is calculated on the design variable space (i.e., $p$ in Eq. (8) is the design variable).

In this work, we independently calculate the $I G D X$ values for two separate PS to confirm whether both PS are obtained simultaneously. The $I G D X$ value for PS1 is denoted as $I G D X_{1}$, while that for PS2 is denoted as $I G D X_{2}$. To assess both PS are equally obtained, we define the difference of two $I G D X$ values as:

$\Delta I G D X=I G D X_{1}-I G D X_{2}$.

If $\triangle I G D X=0$, both PS are equally obtained simultaneously. On the other hand, if $\triangle I G D X<0$, since $I G D X_{1}<I G D X_{2}$, the algorithm is biased to PS1, and vice versa. In the experiment using Bias, the $\triangle I G D X$ value of the synchronous NSGA-III is expected to be 0 , while that of the asynchronous NSGA-III may be less than 0 because its search direction is biased to PS1. We can expect that the proposed method shows similar behavior to the synchronous method by reducing the effect of the evaluation time bias.

We will perform the Kruskal-Wallis test [18] to confirm a statistical difference between three parallelization methods for each criterion. If a significant difference is found with the Kruskal-Wallis test, we perform the posthoc pairwise comparisons using the Wilcoxon rank-sum test [31] with the Bonferroni adjustments [1].

\section{Result}

This section shows the experimental results using MMF with different evaluation time biases. First, we compare the $\triangle I G D X$ values through the number of fitness evaluations to assess whether the proposed method can reduce the effect of evaluation time bias. Then, we confirm the search capability through the number of fitness evaluations by using the $I G D$ value. Finally, we assess the computational efficiency of three parallelization methods by comparing the simulation execution time until obtaining a certain quality of solutions.

\subsection{Effect of evaluation time bias}

Figs. 5 and 6 show the boxplot of the $\Delta I G D X$ value after the maximum number of fitness evaluations. Fig. 5 shows the results using No-bias, while Fig. 6 shows the results using Bias. In these figures, the horizontal axis shows the different parallelization methods, while the vertical axis shows the $\triangle I G D X$ value. The two methods are connected with the "** symbol if a significant difference with a significance level of $5 \%$ is found. While if a significant difference is found with the significance level of $1 \%$, they are marked with the "**" symbol. The dashed line indicates the zero value where $I G D X$ values for PS1 and PS2 are the same. If $\triangle I G D X<0$ (below the dashed line), the solutions close to PS1 (small $x_{2}$ value) are precisely obtained, while $\triangle I G D E>0$ (above the dashed line) indicates the solutions close to PS2 (large $x_{2}$ value) are obtained.

From Fig. 5, when using No-bias, it is first indicated that there is no significant difference in the $\triangle I G D X$ value between the three parallelization methods. In addition, since the $\triangle I G D X$ value is almost zero in all benchmark problems, all parallelization methods can equally search both PS when the evaluation time is not biased.

On the other hand, when using Bias, significant differences are found in MMF2, MMF4, MMF5, and MMF8, while no significant difference is in MMF3 and MMF6. In particular, the asynchronous method acquires 


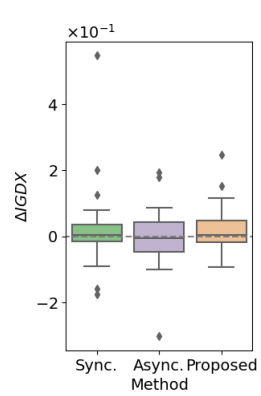

(a) MMF2

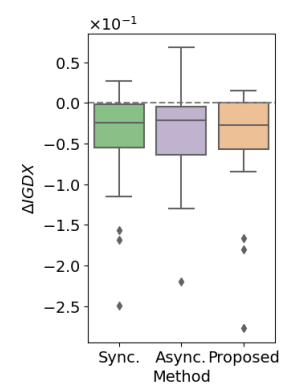

(b) MMF3

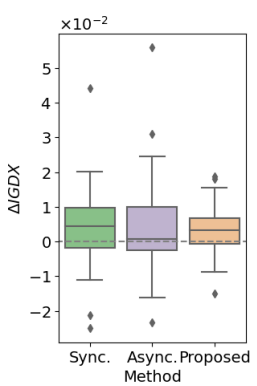

(c) MMF4

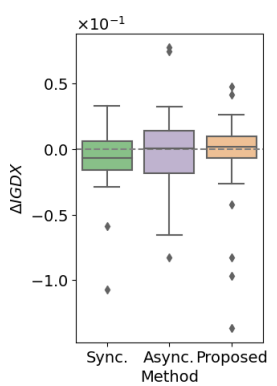

(d) MMF5

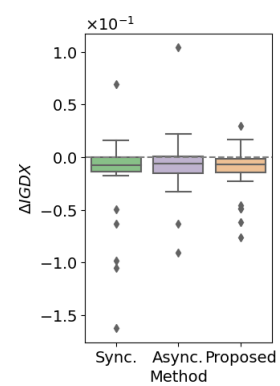

(e) MMF6

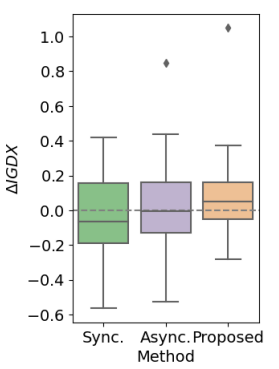

(f) MMF8

Fig. 5: Boxplot of $\triangle I G D X$ with the non-biased evaluation time (No-bias) after the maximum number of fitness evaluations. Comparison of the different parallelization schemes.

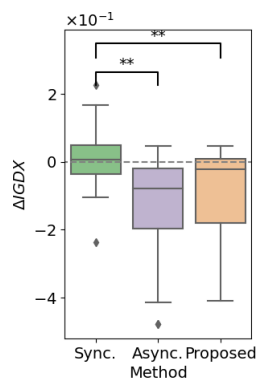

(a) MMF2

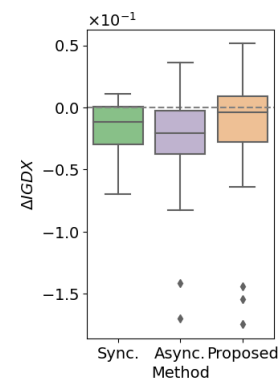

(b) MMF3

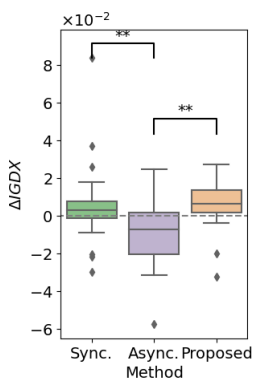

(c) MMF4

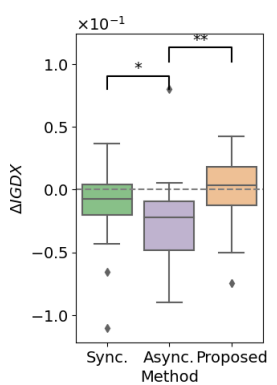

(d) MMF5

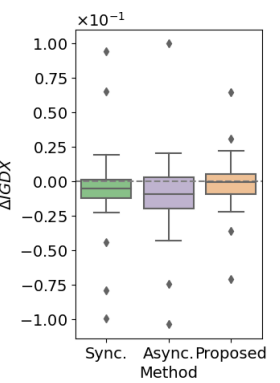

(e) MMF6

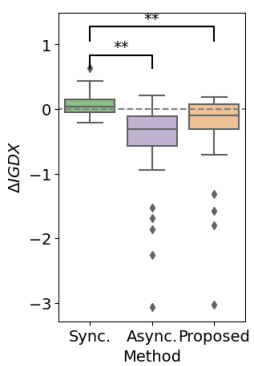

(f) MMF8

Fig. 6: Boxplot of $\triangle I G D X$ with the evaluation time biased for PS1 (Bias) after the maximum number of fitness evaluations. Comparison of the different parallelization schemes.

a significantly small (negative) $\triangle I G D X$ value compared with the synchronous method in MMF2, MMF4, MMF5, and MMF8. This brings out the effect of the evaluation time bias in the asynchronous method.

Focusing on the proposed method, it significantly reduces the effect of the evaluation time bias in MMF4 and MMF5. In these problems, the $\triangle I G D X$ value of the proposed method is not significantly different from that of the synchronous one, while the asynchronous one is significantly biased toward PS1 (shorter evaluation time) compared with the proposed method and the synchronous one. However, in MMF2 and MMF8, the proposed method shows a significantly small (negative) $\triangle I G D X$ value than the synchronous method. At the same time, it is not significantly different from the asynchronous one, though the distribution of the $\triangle I G D X$ value in the proposed method is close to zero compared with the asynchronous one.

These results can be classified into three categories, that is; MMF3 and MMF6, where the $\triangle I G D X$ values are not significantly different between three methods (Proposed $\approx$ Sync. $\approx$ Async.); MMF4 and MMF5, where the asynchronous method is significantly biased toward the region with shorter evaluation value than the others (Proposed $\approx$ Sync. $\gg$ Async.); and MMF2 and MMF8, where the proposed method is also biased (Sync. $\gg$ Proposed $\approx$ Async.).

The difference between these categories can be explained from the perspective of the distribution of PS shown in Fig. 3. First, in MMF3 and MMF6, two PS are overlapped in the $x_{2}$ dimension, and their search is easy to move from one to another. Thus, the effect of the evaluation time bias is small and all methods are not biased. On the other hand, two PS are completely separated in the $x_{2}$ dimension in the other benchmarks, but they are close in MMF4 and MMF5 compared with MMF2 and MMF8. When the regions of PS are separated and their evaluation times are biased, the asynchronous method resulted in the biased search toward regions with short evaluation times. On the other hand, the proposed method can reduce the effect of evaluation time bias even when optimal solutions exist in separate regions with the biased evaluation time and is especially effective for problems where the distance between regions is close (easy transition).

From these results, it is indicated that the proposed method can reduce the effect of the evaluation time bias despite being an asynchronous manner. In contrast, the asynchronous method without the proposed method easy to converge to PS with a shorter evaluation time. 


\subsection{Search capability}

Figs. 7 and 8 show the boxplot of the $I G D$ value after the maximum number of evaluations. Fig. 7 shows the results using No-bias, while Fig. 8 shows the results using Bias. The horizontal axis shows the different parallelization methods, while the vertical axis shows the $I G D$ value. Like the previous results, the two methods are connected with the "*" (5\% of the significance level) or "**" ( $1 \%$ of the significance level) symbols if a significant difference is found.

From Fig. 7, when using the No-bias evaluation time, it is found that there is no significant difference between the three parallelization methods. This indicates that the three methods finally obtain almost the same Pareto fronts. On the other hand, from Fig. 8, it is found that no significant difference is found except for MMF8 when using Bias. In other benchmark problems, the proposed method produces the equivalent $I G D$ value to the other methods. In MMF8, the proposed method obtains a significantly larger (worse) $I G D$ value compared to the asynchronous one. This result can be explained from the result in Fig. 6. Since the asynchronous method is biased toward the search region with a short evaluation time, it precisely obtains the Pareto front by only solutions in PS1. In contrast, since the proposed method and the synchronous method obtain both Pareto sets equally, the $I G D$ values are inferior to those of the asynchronous one that approximates the Pareto front elaborated by PS1 only.

This result indicates that the proposed method does not negatively impact the search capability of the asynchronous method, even though selecting the parents from the limited candidate pool.

\subsection{Computational efficiency}

Figs. 9 and 10 show the elapsed simulation time until obtaining a certain quality of the $I G D$ value. Fig. 9 shows the results using No-bias, while Fig. 10 shows the results using Bias. The horizontal axis shows the different parallelization methods, while the vertical axis shows the elapsed simulation time. Like the previous results, the two methods are connected with the "**" $(5 \%$ of the significance level) or "**" (1\% of the significance level) symbols if a significant difference is found. The target $I G D$ value for each problem is decided from the result in Figs. 7 and 8 as follows:

MMF2: $\quad I G D=2.0 \times 10^{-3}$

MMF3: $I G D=2.0 \times 10^{-3}$

MMF4: $I G D=2.0 \times 10^{-4}$

MMF5: $I G D=5.0 \times 10^{-4}$
MMF6: $I G D=4.0 \times 10^{-4}$

MMF8: $I G D=2.0 \times 10^{-4}$

From Fig. 9, the proposed method significantly reduces the execution time compared with the synchronous one when using Bias. Meanwhile, there is no significant difference between the proposed method and the asynchronous method. This indicates that the proposed method retain the computational efficiency of the asynchronous one.

On the other hand, from Fig. 10, the proposed method also acquires a shorter execution time than the synchronous method. In particular, the proposed method significantly reduces the execution time in MMF4, MMF5, MMF6, and MMF8. In MMF2, MMF3, although no significant difference is found, the proposed method obtains enough better performance in a half execution time compared with the synchronous method. Since the result in Fig. 6 showed the proposed method and the synchronous one obtains both PS with different evaluation times equally, it can be said that the proposed method can simply reduce the execution time while reducing the effect of evaluation time bias.

From the comparison of the proposed and the asynchronous methods, it is indicated that the proposed method requires a significantly longer execution time when using Bias. However, this is because the asynchronous method is biased toward searching for solutions with shorter evaluation times (PS1), as indicated in Fig. 6. On the other hand, since the proposed method obtains solutions with longer evaluation times, its execution time increases from the asynchronous one.

This result indicates that the proposed method retains the computational efficiency of the asynchronous method while avoiding being biased toward a search region with a short evaluation time.

\subsection{Summary}

Finally, this subsection summarizes the experimental results shown in this section. We compared three parallelization methods on biased and non-biased evaluation time. First, it was confirmed that the proposed method could reduce the negative influence of the evaluation time bias, in contrast to the asynchronous one in which the search direction is biased toward the search region with a shorter evaluation time. From the viewpoint of the search capability, these three methods almost achieve the equivalent performance on both biased and non-biased evaluation time. This indicates that the proposed method does not hurt the search capability. Finally, the proposed method can significantly reduce the execution time when the evaluation times of so- 


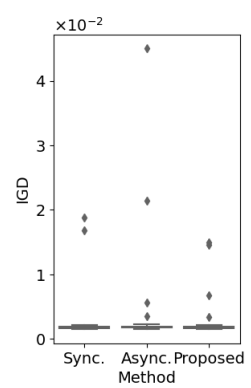

(a) MMF2

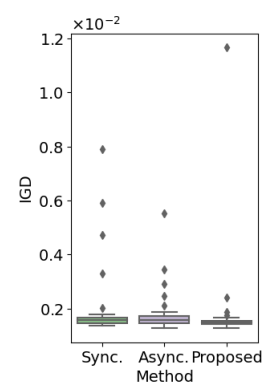

(b) MMF3

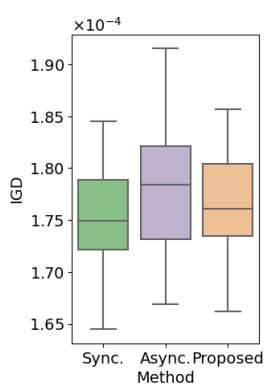

(c) MMF4

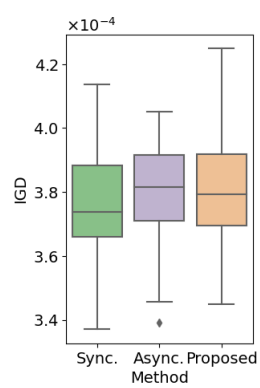

(d) MMF5

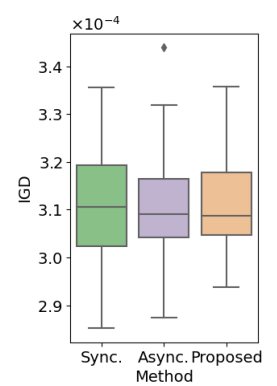

(e) MMF6

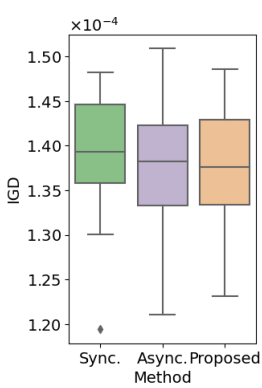

(f) $\mathrm{MMF} 8$

Fig. 7: Boxplot of IGD with the non-biased evaluation time (No-bias) after the maximum number of fitness evaluations. Comparison of the different parallelization schemes.

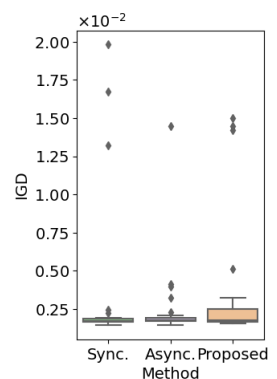

(a) MMF2

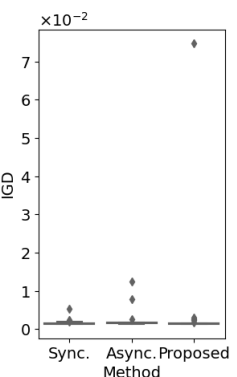

(b) MMF3

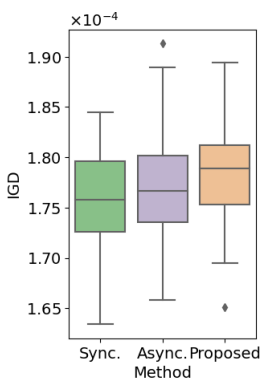

(c) MMF4

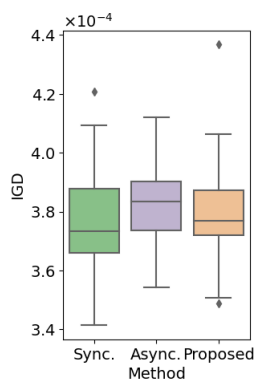

(d) MMF5

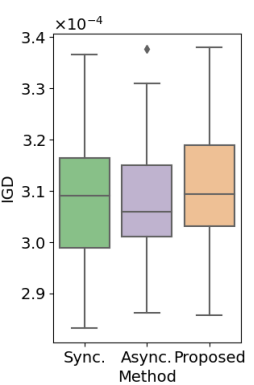

(e) MMF6

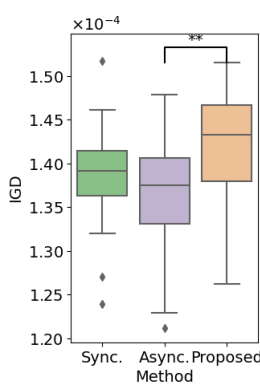

(f) MMF8

Fig. 8: Boxplot of IGD with the evaluation time biased for PS1 (Bias) after the maximum number of fitness evaluations. Comparison of the different parallelization schemes.

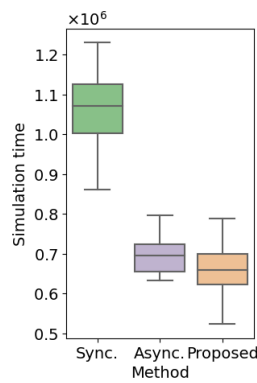

(a) MMF2

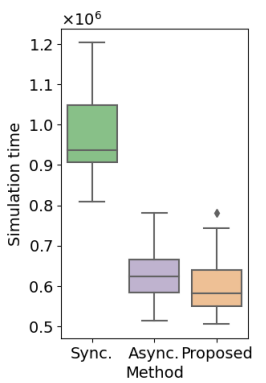

(b) MMF3

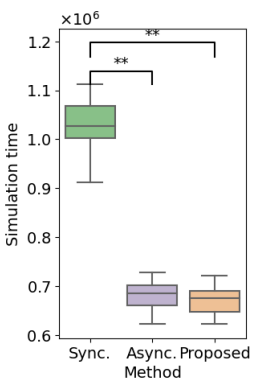

(c) MMF4

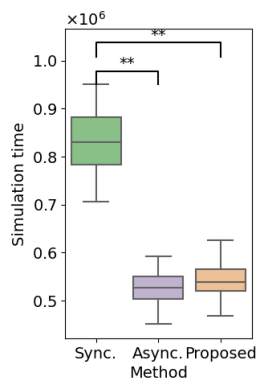

(d) MMF5

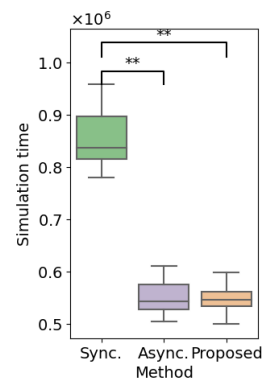

(e) MMF6

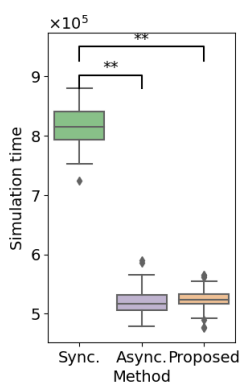

(f) $M M F 8$

Fig. 9: Boxplot of the simulation time until reaching a certain $I G D$ value the non-biased evaluation time (No-bias). Comparison of the different parallelization schemes.

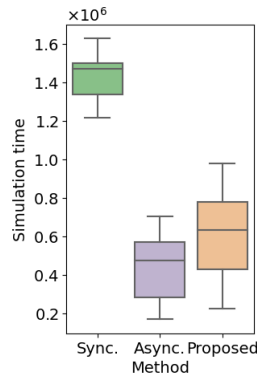

(a) MMF2

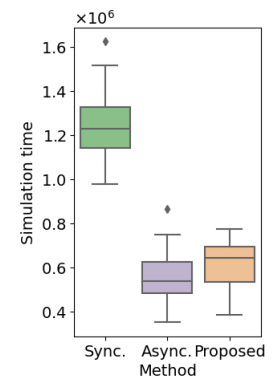

(b) MMF3

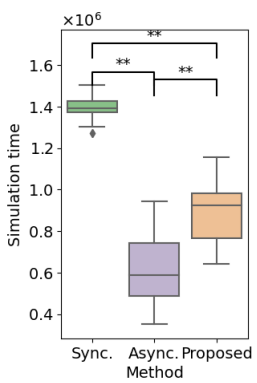

(c) MMF4

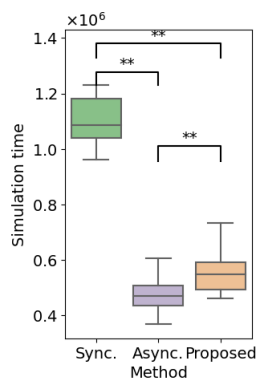

(d) MMF5

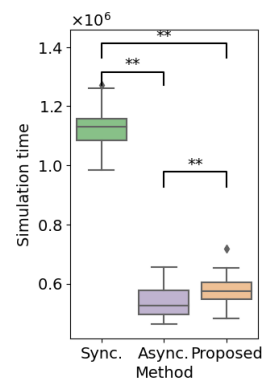

(e) MMF6

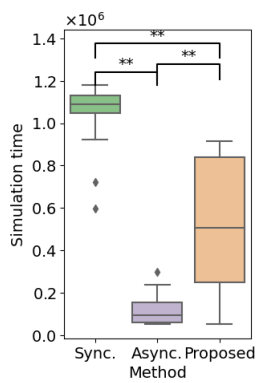

(f) MMF8

Fig. 10: Boxplot of the simulation time until reaching a certain IGD value the evaluation time biased for PS1 (Bias). Comparison of the different parallelization schemes. 
lutions differ from the synchronous method while retaining the search capability of the synchronous one. Totally, the proposed method possesses high search capability and high computational efficiency for the problem with heterogeneous (both biased and non-biased) evaluation time.

\section{Comparison of Selection Ratio}

This section investigates how the selection ratio of the proposed method ( $r_{s}$ in Algorithm 2) affects the search capability and computational efficiency. Although we used $r_{s}=0.5$ in the previous experiments, we compare $r_{s}=\{0.1,0.3,0.5,0.7,0.9\}$. In the following subsections, we first show the effect of the evaluation time bias and then show the results from the viewpoints of the search capability and the computational efficiency as same as the previous section.

\subsection{Effect of evaluation time bias}

Figs. 11 and 12 show the boxplot of the $\triangle I G D X$ value to confirm the influence of the evaluation time bias. Fig. 11 shows the results using No-bias, while Fig. 12 shows the results using Bias. The horizontal axis shows the selection ratio $r_{s}$, while the vertical axis shows the $\triangle I G D X$ value. If there exists a significant difference with the significance level of $5 \%$ between the two parameters, they are connected with a line marked with the "*" symbol. While if a significant difference is found with the significance level of $1 \%$, they are marked with the "**" symbol.

First, from Fig. 11, it is confirmed that there is no significant difference between different selection ratios in the non-biased evaluation time. On the other hand, we can find a significant impact of the selection ratio when using the biased evaluation time as shown in Fig. 12. In concrete, we can find a tendency that the $\triangle I G D X$ value decreases as the selection ratio $r_{s}$ increases. Specifically, the selection ratio of $r_{s}=0.9$ is significantly biased toward the search region with a shorter evaluation time (a negative $\triangle I G D X$ value) compared with the other selection ratios. Since the proposed method with a large selection ratio selects parent individuals from a large candidate pool that contains solutions with a large search frequency, it gets close to the asynchronous method without the proposed method. Therefore, the larger the selection ratio $r_{s}$, the more susceptible the evaluation time bias. From this result, it is indicated that a large selection ratio should be avoided to prevent from being affected by the evaluation time bias.

\subsection{Search capability}

Figs. 13 and 14 show the boxplot of the $I G D$ value after the maximum number of evaluations. Fig. 13 shows the results using No-bias, while Fig. 14 shows the results using Bias. The horizontal axis shows the selection ratio $r_{s}$, while the vertical axis shows the $I G D$ value. Like above, the two parameters are connected with the "** or "**" symbols if a significant difference is found.

From these results, we can find that the result with $r_{s}=0.1$ shows a significantly worse $I G D$ value between five different selection ratios in some problems. This indicates that the small $r_{s}$ shows a negative influence on the search capability. This happens in both the non-biased and biased evaluation times. When setting $r_{s}=0.1$, the proposed method selects parents from $10 \%$ of solutions in the current population, and this restricts the diversity of the parent solutions and reduces the search capability. On the other hand, the results with $r_{s} \geq 0.3$ show almost the same performance in all problems and evaluation times. This result indicates that a small selection ratio should be avoided in terms of the search capability.

\subsection{Computational efficiency}

Figs. 15 and 16 show the simulation time until reaching a certain $I G D$ value. Fig. 15 shows the results using No-bias, while Fig. 16 shows the results with Bias. The horizontal axis shows the selection ratio, while the vertical axis shows the simulation time. The target $I G D$ value is same as Section 6.3. Like above, the two parameters with the significant difference are connected with the "**" (5\% of the significance level) and "**" (1\% of the significance level) symbols.

First, from Fig. 15 where No-bias is used, a significant difference is found in MMF6. In this problem, the selection ratio $r_{s}=0.1$ requires a significantly longer simulation time than the others. As shown in Fig. 11, all selection ratios can equally obtain both PS in No-bias. Hence, this is caused by the lower search capability of the selection ratio $r_{s}=0.1$, which was demonstrated in Fig. 13.

From Fig. 16, on the other hand, the larger the selection ratio is used, the shorter the simulation time is obtained. Here, we need to consider the effect of the evaluation time bias on the execution time. Specifically, when using the selection ratios of $r_{s}=0.7,0.9$, the search direction is biased toward a region with a shorter evaluation time, resulting in a shorter execution time. In fact, from these results, we can see that the evaluation time of the selection ratios of $r_{s}=0.9$ is 


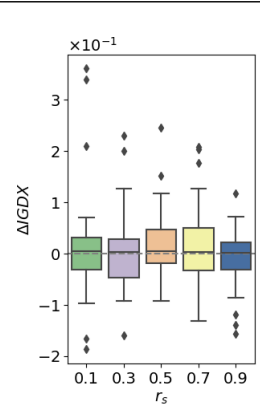

(a) MMF2

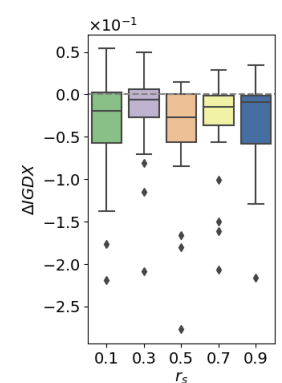

(b) MMF3

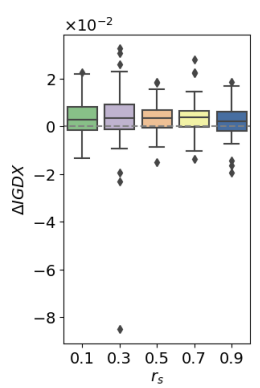

(c) MMF4

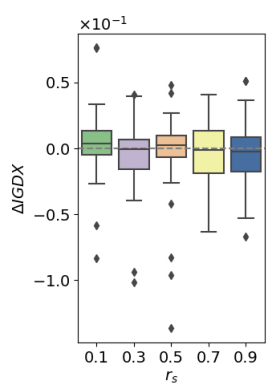

(d) MMF5

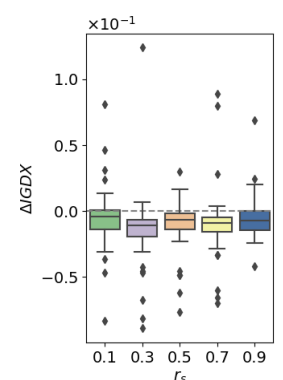

(e) MMF6

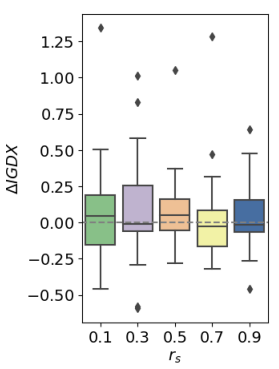

(f) MMF8

Fig. 11: Boxplot of $\triangle I G D X$ with the non-biased evaluation time bias (No-bias) after the maximum number of fitness evaluations. Comparison of five selection ratios.

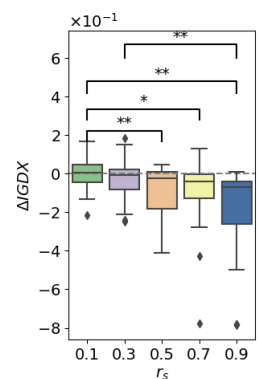

(a) MMF2

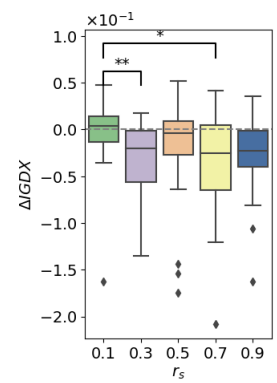

(b) MMF3

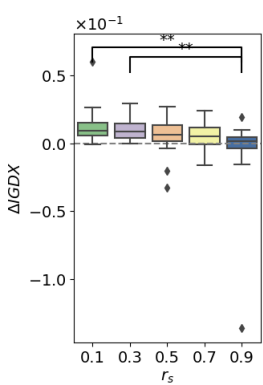

(c) MMF4

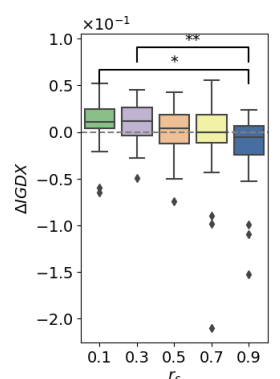

(d) MMF5

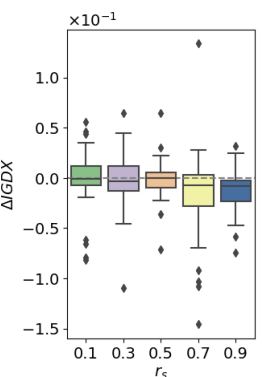

(e) MMF6

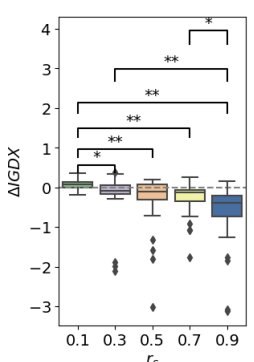

(f) $\mathrm{MMF} 8$

Fig. 12: Boxplot of $\triangle I G D X$ with the evaluation time biased for PS1 (Bias) after the maximum number of fitness evaluations. Comparison of five selection ratios.

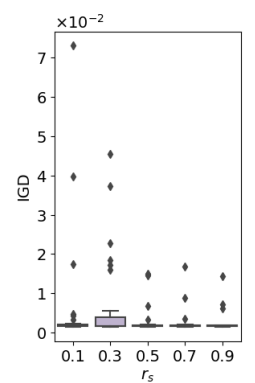

(a) MMF2

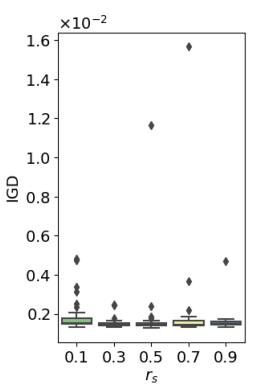

(b) MMF3

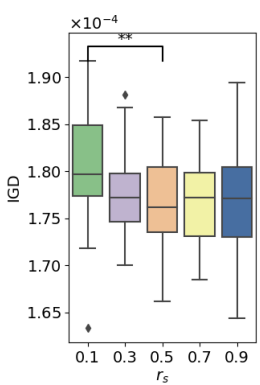

(c) MMF4

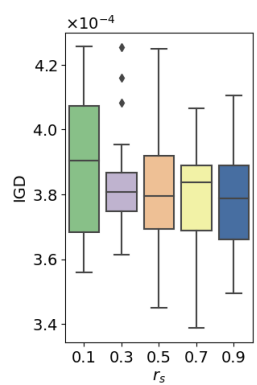

(d) MMF5

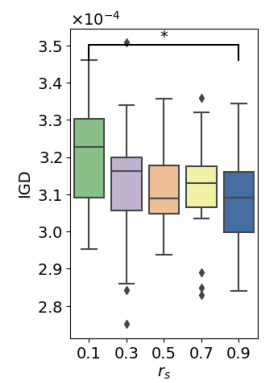

(e) MMF6

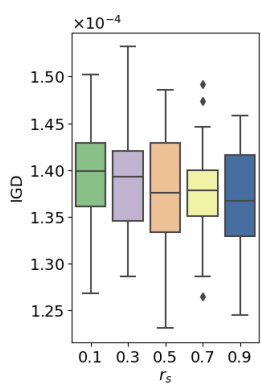

(f) MMF8

Fig. 13: Boxplot of $I G D$ with the non-biased evaluation time (No-bias) after the maximum number of fitness evaluations. Comparison of five selection ratios.

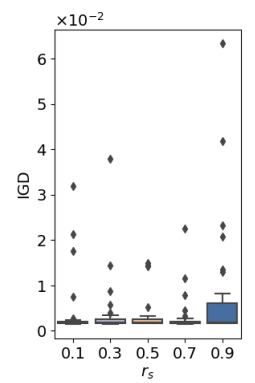

(a) MMF2

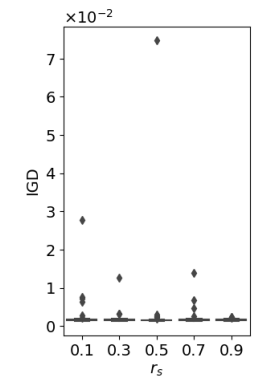

(b) MMF3

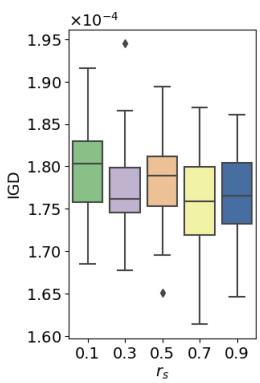

(c) MMF4

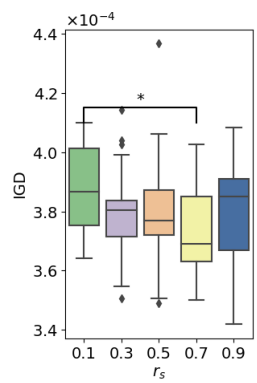

(d) MMF5

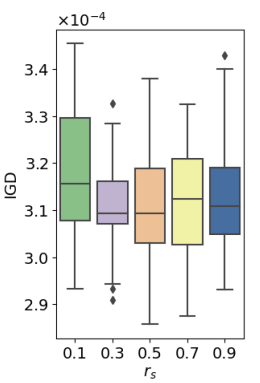

(e) MMF6

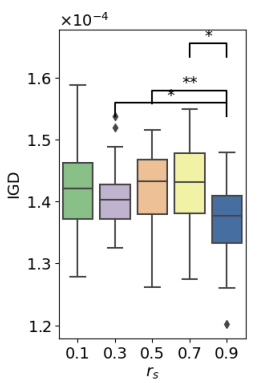

(f) MMF8

Fig. 14: Boxplot of IGD with the evaluation time biased for PS1 (Bias) after the maximum number of fitness evaluations. Comparison of five selection ratios. 


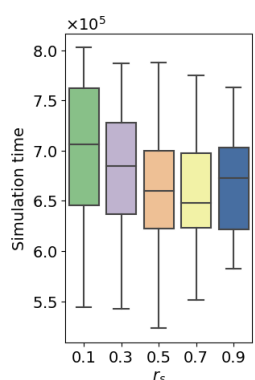

(a) MMF2

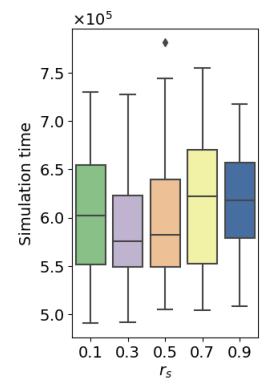

(b) MMF3

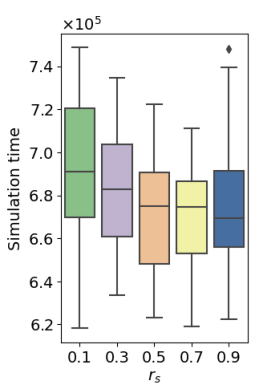

(c) MMF4

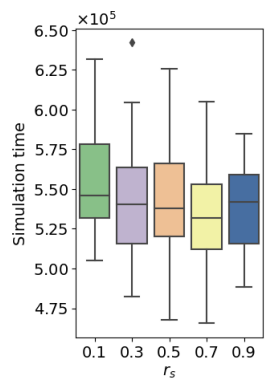

(d) MMF5

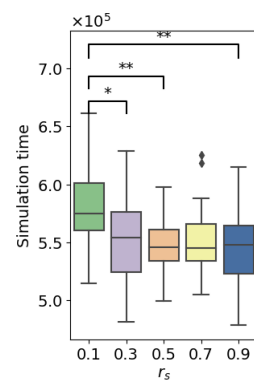

(e) MMF6

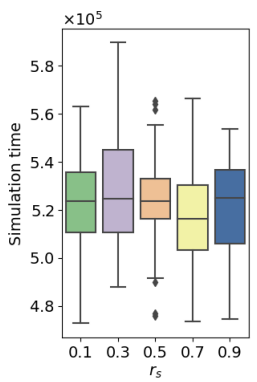

(f) MMF8

Fig. 15: Boxplot of the simulation time until reaching a certain $I G D$ value the non evaluation time bias (No-bias). Comparison of five selection ratios.

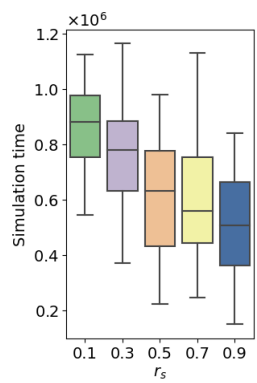

(a) MMF2

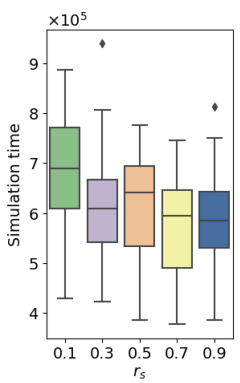

(b) MMF3

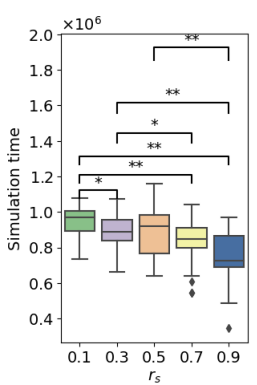

(c) MMF4

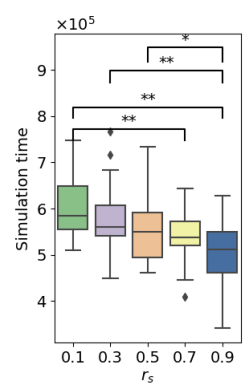

(d) MMF5

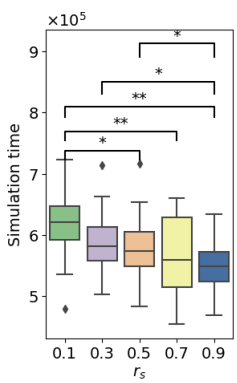

(e) MMF6

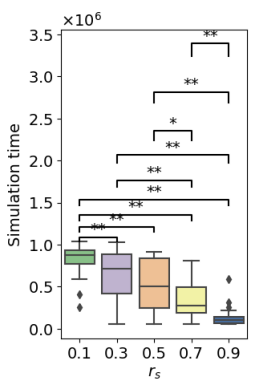

(f) $\mathrm{MMF} 8$

Fig. 16: Boxplot of the simulation time until reaching a certain $I G D$ value the evaluation time biased for PS1 (Bias). Comparison of five selection ratios.

the shortest in all problems, and some are significantly shorter than the others.

For the other selection ratios, the selection ratio of $r_{s}=0.1$ obtains the significantly longest execution time in MMF4, MMF5, MMF6, and MMF8. This is due to a combination of the facts that the small selection ratio decreases its search capability (shown in Section 7.2), and it obtains solutions with both longer and shorter evaluation times by reducing the effect of the evaluation time bias (shown in Section 7.1).

In contrast, the selection ratios of $r_{s}=0.3,0.5 \mathrm{ac}-$ quire stably shorter execution times in both evaluation time settings. Since these two ratios acquire competitive search capability to the large selection ratios (e.g., $\left.r_{s}=0.7,0.9\right)$ and decrease the effect of the evaluation time bias to the same extent as using smaller ratios (e.g., $r_{s}=0.1$ ), we can say that such selection ratios are appropriate for the proposed method.

\subsection{Summary}

This subsection summarizes the experimental results of the comparison in the selection ratios.

First, from the viewpoint of the effect of the evaluation time bias, a large selection ratio should not be employed because it is highly affected by the evaluation time bias. Second, from the viewpoint of the search capability, a small selection ratio should be avoided because it decreases the search capability by limiting parent candidates in surplus. Finally, from the viewpoint of the computational efficiency, although the execution time varies depends on the selection ratio, the medium selection ratio such as $r_{s}=0.3,0.5$ shows the stably high computational efficiency.

From these results, it can be suggested that the medium selection ratio such as $r_{s}=0.3,0.5$ should be chosen for the proposed selection method to take balance between the search capability and the computational efficiency while reducing the effect of the evaluation time bias. This paper recommends using the selection ratio of $0.3 \leq r_{s} \leq 0.5$ as the conclusion.

\section{Conclusion}

This paper proposed a new parent selection strategy for reducing the effect of evaluation time bias in APEAs. In particular, the proposed method introduces a new parameter to retain the search frequency of each solution and performs the parent selection from the pre-selected candidate pool according to this parameter. By selecting parents to make the search frequency of solutions 
in the population uniform, the proposed method prevents an APEA from being biased toward the search region with a short evaluation time. This paper conducted an experiment to investigate the effectiveness of the proposed method on multi-objective optimization problems. We used toy problems designed based on the MMF benchmarks for the detailed analysis of the evaluation time bias in the experiments. The proposed method was applied to the asynchronous parallel NSGA-III and was compared with the synchronous and the asynchronous NSGA-III.

The experimental results indicated that the proposed method could reduce the negative influence of the evaluation time bias, in contrast to APEAs in which the search direction is biased toward the search region of a shorter evaluation time. The proposed method also does not hurt the search capability of APEAs while reducing the execution time significantly from SPEAs. This result revealed that the proposed method possesses high search capability and high computational efficiency for the problems with heterogeneous evaluation time.

We additionally analyzed the impact of the selection ratio in the proposed method using the same toy problems. This analysis indicated that the medium selection ratio in $0.3 \leq r_{s} \leq 0.5$ acquires an appropriate balance between the search capability and the computational efficiency while reducing the effect of the evaluation time bias.

In the near future, it should be addressed to further analyze the proposed method on other benchmarks and with other EA methods. In addition, although this paper only compared the proposed method with the synchronous and the asynchronous method, it should be compared with or integrated into a semi-asynchronous method $[15,16]$ to adapt any characteristics of the evaluation time.

Acknowledgements This work was supported by Japan Society for the Promotion of Science Grant-in-Aid for Young Scientists Grant Number JP19K20362.

\section{Conflict of interest}

The author declares that they have no conflict of interest.

\section{A Definition of MMF}

This appendix provides concrete definitions of MMF functions. Table 3 shows the definitions of MMF functions used in this work. In this table, "PS" indicates the distribution of the Pareto set, while "PF" indicates the distribution of the Pareto front for each problem. Fig. 17, on the other hand, shows the distribution of the Pareto front on bi-objective space. In this figure, the horizontal axis shows the first objective function value $\left(f_{1}\right)$, while the vertical axis shows the second objective $\left(f_{2}\right)$. These definitions basically come from [33], but more thorough descriptions can be found in [19].

\section{References}

1. Abdi, H., et al.: Bonferroni and šidák corrections for multiple comparisons. Encyclopedia of measurement and statistics 3, 103-107 (2007)

2. Alba, E., Luque, G., Nesmachnow, S.: Parallel metaheuristics: recent advances and new trends. International Transactions in Operational Research 20(1), 1-48 (2013). DOI 10.1111/j.1475-3995.2012.00862. x. URL https://onlinelibrary.wiley.com/doi/abs/ $10.1111 / j .1475-3995.2012 .00862 . x$

3. Alba, E., Tomassini, M.: Parallelism and evolutionary algorithms. IEEE Transactions on Evolutionary Computation 6(5), 443-462 (2002). DOI 10.1109/TEVC.2002. 800880

4. Coello, C.A.C., Cortés, N.C.: Solving multiobjective optimization problems using an artificial immune system. Genetic Programming and Evolvable Machines 6(2), 163190 (2005). DOI 10.1007/s10710-005-6164-x. URL https://doi.org/10.1007/s10710-005-6164-x

5. Deb, K., Agrawal, R.B.: Simulated Binary Crossover for Continuous Search Space. Complex Systems 9, 115-148 (1995). URL citeseer.ist.psu.edu/deb95simulated. html

6. Deb, K., Goyal, M.: A combined genetic adaptive search (geneas) for engineering design. Computer Science and Informatics 26, 30-45 (1996)

7. Deb, K., Jain, H.: An evolutionary many-objective optimization algorithm using reference-point-based nondominated sorting approach, part i: Solving problems with box constraints. IEEE Transactions on Evolutionary Computation 18(4), 577-601 (2014). DOI 10.1109/TEVC.2013. 2281535

8. Depolli, M., Trobec, R., Filipic, B.: Asynchronous master-slave parallelization of differential evolution for multi-objective optimization. Evolutionary Computation $\mathbf{2 1}(2), 261-291$ (2013)

9. Durillo, J.J., Nebro, A.J., Luna, F., Alba, E.: A study of master-slave approaches to parallelize nsga-ii. In: 2008 IEEE International Symposium on Parallel and Distributed Processing, pp. 1-8 (2008). DOI 10.1109/ IPDPS.2008.4536375

10. Harada, T.: Mathematical model of asynchronous parallel evolutionary algorithm to analyze influence of evaluation time bias. In: 2019 IEEE Asia-Pacific Conference on Computer Science and Data Engineering (CSDE), pp. 1-8 (2019). DOI 10.1109/CSDE48274.2019.9162360

11. Harada, T.: Search progress dependent parent selection for avoiding evaluation time bias in asynchronous parallel multi-objective evolutionary algorithms. In: 2020 IEEE Symposium Series on Computational Intelligence (SSCI), pp. 1013-1020 (2020). DOI 10.1109/SSCI47803. 2020.9308152

12. Harada, T., Alba, E.: Parallel genetic algorithms: A useful survey. ACM Computing Surveys 53(4) (2020). DOI 10.1145/3400031. URL https://doi.org/10.1145/ 3400031

13. Harada, T., Takadama, K.: Asynchronous evaluation based genetic programming: Comparison of asynchronous 
Table 3: Definitions of MMF functions $[19,33]$

\begin{tabular}{|c|c|}
\hline Problem & Objectives, domain, Pareto set (PS), and Pareto front (PF) \\
\hline MMF2 & $\begin{array}{l}f_{1}=x_{1} \\
f_{2}= \begin{cases}1-\sqrt{x_{1}}+2\left(4\left(x_{2}-\sqrt{x_{1}}\right)^{2}-2 \cos \left(\frac{20\left(x_{2}-\sqrt{x_{1}}\right) \pi}{\sqrt{2}}\right)+2\right) \\
1-\sqrt{x_{1}}+2\left(4\left(x_{2}-1-\sqrt{x_{1}}\right)^{2}-2 \cos \left(\frac{20\left(x_{2}-1-\sqrt{x_{1}}\right) \pi}{\sqrt{2}}\right)+2\right) & 0 \leq x_{2} \leq 1 \\
1 \leq x_{2} \leq 2\end{cases} \\
0 \leq x_{1} \leq 1,0 \leq x_{2} \leq 2 \\
\text { PS: } x_{1}=\left\{\begin{array}{ll}x_{2}^{2} & 0 \leq x_{2} \leq 1 \\
\left(x_{2}-1\right)^{2} & 1 \leq x_{2} \leq 2\end{array}, \text { PF: } f_{2}=1-\sqrt{f_{1}}, 0 \leq f_{1} \leq 1\right.\end{array}$ \\
\hline MMF3 & 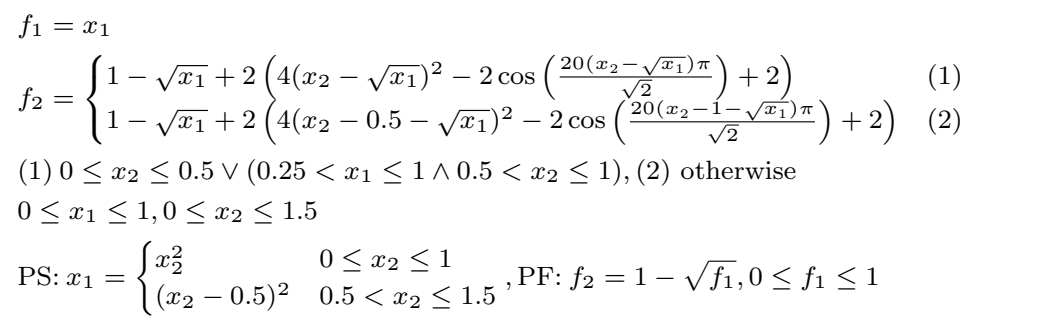 \\
\hline MMF4 & $\begin{array}{l}f_{1}=\left|x_{1}\right| \\
f_{2}= \begin{cases}1-x_{1}^{2}+2\left(x_{2}-\sin \left(\pi\left|x_{1}\right|\right)\right)^{2} & 0 \leq x_{2}<1 \\
1-x_{1}^{2}+2\left(x_{2}-1-\sin \left(\pi\left|x_{1}\right|\right)\right)^{2} & 1 \leq x_{2} \leq 2\end{cases} \\
-1 \leq x_{1} \leq 1,0 \leq x_{2} \leq 2 \\
\text { PS: } x_{2}=\left\{\begin{array}{ll}\sin \left(\pi\left|x_{1}\right|\right) & 0 \leq x_{2} \leq 1 \\
\sin \left(\pi\left|x_{1}\right|\right)+1 & 1<x_{2} \leq 2\end{array}, \text { PF: } f_{2}=1-f_{1}^{2}, 0 \leq f_{1} \leq 1\right.\end{array}$ \\
\hline MMF5 & $\begin{array}{l}f_{1}=\left|x_{1}-2\right| \\
f_{2}= \begin{cases}1-\sqrt{\left|x_{1}-2\right|}+2\left(x_{2}-\sin \left(6 \pi\left|x_{1}-2\right|+\pi\right)\right)^{2} & -1 \leq x_{2}<1 \\
1-\sqrt{\left|x_{1}-2\right|}+2\left(x_{2}-2-\sin \left(6 \pi\left|x_{1}-2\right|+\pi\right)\right)^{2} & 1<x_{2}<3\end{cases} \\
-1 \leq x_{1} \leq 3,1 \leq x_{2} \leq 3 \\
\text { PS: } x_{2}=\left\{\begin{array}{ll}\sin \left(6 \pi\left|x_{1}-2\right|+\pi\right) \\
\sin \left(6 \pi\left|x_{1}-2\right|+\pi\right)+2\end{array} \quad, \text { PF: } f_{2}=1-\sqrt{f_{1}}, 0 \leq f_{1} \leq 1\right.\end{array}$ \\
\hline MMF6 & $\begin{array}{l}f_{1}=\left|x_{1}-2\right| \\
f_{2}= \begin{cases}1-\sqrt{\left|x_{1}-2\right|}+2\left(x_{2}-\sin \left(6 \pi\left|x_{1}-2\right|+\pi\right)\right)^{2} & -1 \leq x_{2}<1 \\
1-\sqrt{\left|x_{1}-2\right|}+2\left(x_{2}-1-\sin \left(6 \pi\left|x_{1}-2\right|+\pi\right)\right)^{2} & 1<x_{2}<3\end{cases} \\
-1 \leq x_{1} \leq 3,1 \leq x_{2} \leq 2 \\
\text { PS: } x_{2}=\left\{\begin{array}{ll}\sin \left(6 \pi\left|x_{1}-2\right|+\pi\right) \\
\sin \left(6 \pi\left|x_{1}-2\right|+\pi\right)+1\end{array} \quad, \text { PF: } f_{2}=1-\sqrt{f_{1}}, 0 \leq f_{1} \leq 1\right.\end{array}$ \\
\hline MMF8 & $\begin{array}{l}f_{1}=\sin \left|x_{1}\right| \\
f_{2}= \begin{cases}\sqrt{1-\sin \left(\left|x_{1}\right|\right)^{2}}+2\left(x_{2}-\sin \left(\left|x_{1}\right|\right)-\left|x_{1}\right|\right)^{2} & 0 \leq x_{2}<4 \\
\sqrt{1-\sin \left(\left|x_{1}\right|\right)^{2}}+2\left(x_{2}-4-\sin \left(\left|x_{1}\right|\right)-\left|x_{1}\right|\right)^{2} & 4<x_{2} \leq 9\end{cases} \\
-\pi \leq x_{1} \leq \pi, 0 \leq x_{2} \leq 9 \\
\text { PS: } x_{2}=\left\{\begin{array}{ll}\sin \left(\left|x_{1}\right|\right)+\left|x_{1}\right| & 0 \leq x_{2} \leq 4 \\
\sin \left(\left|x_{1}\right|\right)+\left|x_{1}\right|+4 & 4<x_{2} \leq 9\end{array}, \text { PF: } f_{2}=\sqrt{1-f_{1}^{2}}, 0 \leq f_{1} \leq 1\right.\end{array}$ \\
\hline
\end{tabular}

and synchronous evaluation and its analysis. In: K. Krawiec, A. Moraglio, T. Hu, A. Etaner-Uyar, B. Hu (eds.) Genetic Programming, Lecture Notes in Computer Science, vol. 7831, pp. 241-252. Springer Berlin Heidelberg (2013). DOI 10.1007/978-3-642-37207-0_21. URL http://dx.doi.org/10.1007/978-3-642-37207-0_21

14. Harada, T., Takadama, K.: Asynchronously evolving solutions with excessively different evaluation time by reference-based evaluation. In: GECCO '14: Proceedings of the 2014 conference on Genetic and evolutionary computation, pp. 911-918. ACM, Vancouver, BC, Canada (2014). DOI doi:10.1145/2576768.2598330. URL http://doi.acm.org/10.1145/2576768.2598330

15. Harada, T., Takadama, K.: Performance Comparison of Parallel Asynchronous Multi-Objective Evolutionary Algorithm with Different Asynchrony. In: Proceedings of 


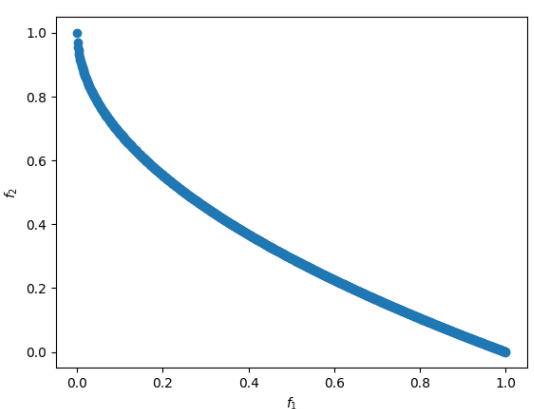

(a) MMF2

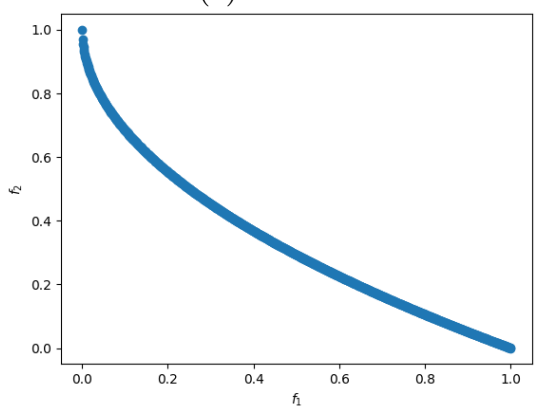

(d) MMF5

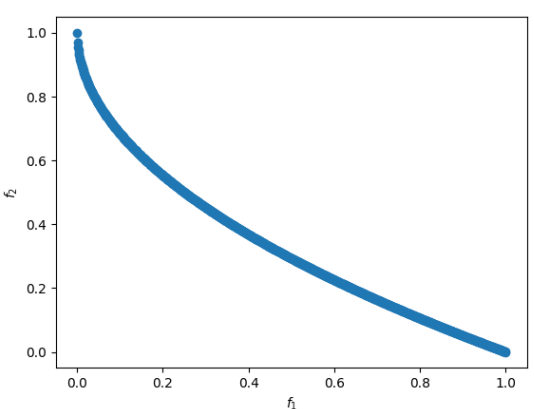

(b) MMF3

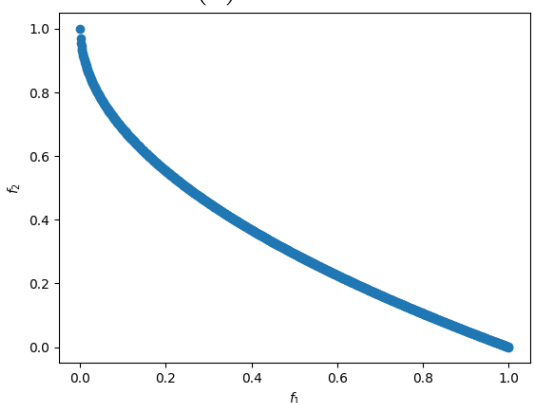

(e) MMF6

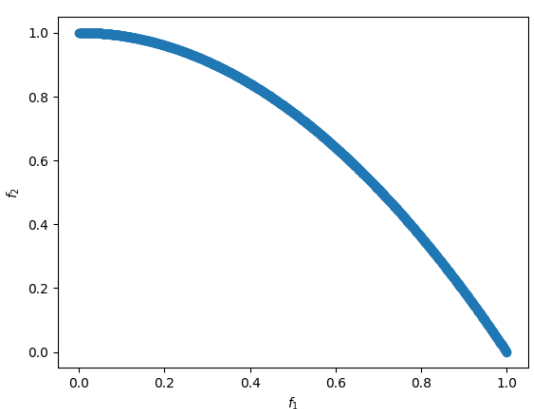

(c) MMF4

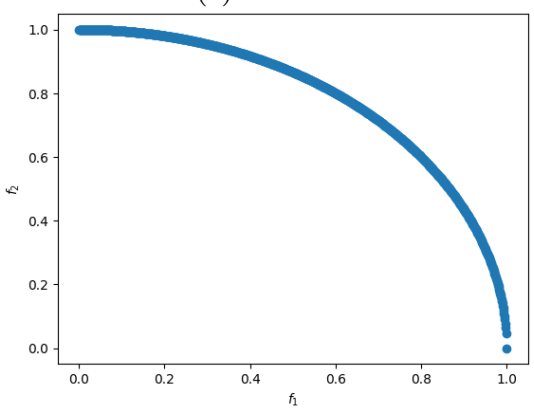

(f) MMF8

Fig. 17: The distribution of Pareto front (PF) in MMFs used in this work

the 2017 IEEE Congress on Evolutionary Computation (CEC 2017), pp. 1215-1222 (2017). DOI 10.1109/CEC. 2017.7969444

16. Harada, T., Takadama, K.: Analysis of semiasynchronous multi-objective evolutionary algorithm with different asynchronies. Soft Computing 24(4), 2917-2939 (2020). DOI 10.1007/s00500-019-04071-7. URL https://doi.org/10.1007/s00500-019-04071-7

17. Koh, B.I., George, A.D., Haftka, R.T., Fregly, B.J.: Parallel asynchronous particle swarm optimization. International Journal for Numerical Methods in Engineering 67(4), 578-595 (2006). DOI 10.1002/ nme.1646. URL http://www.ncbi.nlm.nih.gov/pmc/ articles/PMC1769316/

18. Kruskal, W.H., Wallis, W.A.: Use of Ranks in OneCriterion Variance Analysis. Journal of the American Statistical Association 47(260), 583-621 (1952). DOI 10.2307/2280779. URL http://dx.doi.org/10.2307/ 2280779

19. Liang, J., Xu, W., Yue, C., Yu, K., Song, H., Crisalle, O.D., Qu, B.: Multimodal multiobjective optimization with differential evolution. Swarm and Evolutionary Computation 44, 1028-1059 (2019). DOI https://doi.org/10.1016/j.swevo.2018.10.016. URL https://www.sciencedirect.com/science/article/ pii/S2210650217308362

20. Luna, F., Zavala, G.R., Nebro, A.J., Durillo, J.J., Coello, C.A.C.: Distributed multi-objective metaheuristics for real-world structural optimization problems. The Computer Journal 59(6), 777-792 (2016). DOI 10.1093/comjnl/bxu082. URL http://dx.doi.org/10. 1093/comjnl/bxu082

21. Noguchi, H., Harada, T., Thawonmas, R.: Parallel differential evolution applied to interleaving generation with precedence evaluation of tentative solutions. In: Proceedings of the Genetic and Evolutionary Computation Con- ference, GECCO '21, p. 706-713. Association for Computing Machinery, New York, NY, USA (2021). DOI 10.1145/3449639.3459337. URL https://doi.org/10. $1145 / 3449639.3459337$

22. Noguchi, H., Sonoda, A., Harada, T., Thawonmas, R.: Interleaving generation evolutionary algorithm with precedence evaluation of tentative offspring. In: 2020 59th Annual Conference of the Society of Instrument and Control Engineers of Japan (SICE), pp. 832-837 (2020). DOI 10.23919/SICE48898.2020.9240410

23. Oyama, A., Kohira, T., Kemmotsu, H., Tatsukawa, T., Watanabe, T.: Simultaneous structure design optimization of multiple car models using the k computer. In: 2017 IEEE Symposium Series on Computational Intelligence (SSCI), pp. 1-4 (2017). DOI 10.1109/SSCI.2017.8285350

24. Pilát, M., Neruda, R.: Parallel evolutionary algorithm with interleaving generations. In: Proceedings of the Genetic and Evolutionary Computation Conference, GECCO '17, p. 865-872. Association for Computing Machinery, New York, NY, USA (2017). DOI 10.1145/3071178.3071309. URL https://doi.org/10. $1145 / 3071178.3071309$

25. Sahingoz, O.K.: Generation of bezier curve-based flyable trajectories for multi-uav systems with parallel genetic algorithm. Journal of Intelligent \& Robotic Systems 74(1), 499-511 (2014). DOI 10.1007/s10846-013-9968-6. URL https://doi.org/10.1007/s10846-013-9968-6

26. Scott, E.O., De Jong, K.A.: Evaluation-time bias in asynchronous evolutionary algorithms. In: Proceedings of the Companion Publication of the 2015 Annual Conference on Genetic and Evolutionary Computation, GECCO Companion '15, pp. 1209-1212. ACM, New York, NY, USA (2015). DOI 10.1145/2739482.2768482. URL http: //doi.acm.org/10.1145/2739482.2768482

27. Scott, E.O., De Jong, K.A.: Understanding simple asynchronous evolutionary algorithms. In: Proceedings of the 
2015 ACM Conference on Foundations of Genetic Algorithms XIII, FOGA '15, pp. 85-98. ACM, New York, NY, USA (2015). DOI 10.1145/2725494.2725509. URL http://doi.acm.org/10.1145/2725494.2725509

28. Shayeghi, A., Gotz, D., Davis, J.B.A., Schafer, R., Johnston, R.L.: Pool-bcga: a parallelised generation-free genetic algorithm for the ab initio global optimisation of nanoalloy clusters. Phys. Chem. Chem. Phys. 17, 21042112 (2015). DOI 10.1039/C4CP04323E. URL http: //dx.doi.org/10.1039/C4CP04323E

29. Soufan, O., Kleftogiannis, D., Kalnis, P., Bajic, V.B.: Dwfs: A wrapper feature selection tool based on a parallel genetic algorithm. PLOS ONE 10(2), 1-23 (2015). DOI 10.1371/journal.pone.0117988. URL https://doi. org/10.1371/journal . pone.0117988

30. Wessing, S., Rudolph, G., Menges, D.A.: Comparing asynchronous and synchronous parallelization of the smsemoa. In: J. Handl, E. Hart, P.R. Lewis, M. LópezIbáñez, G. Ochoa, B. Paechter (eds.) Parallel Problem Solving from Nature - PPSN XIV, pp. 558-567. Springer International Publishing, Cham (2016)

31. Wilson, S.W.: Explore/Exploit Strategies in Autonomy. In: P. Maes, M. Mataric, J.A. Meyer, J. Pollack, S. Wilson (eds.) From Animals to Animats 4 Proceedings of the Fourth International Conference on Simulation of Adaptive Behavior, pp. 325-332. MIT Press (1996). URL http://citeseer.ist.psu.edu/107367.html

32. Ye, C.J., Huang, M.X.: Multi-objective optimal power flow considering transient stability based on parallel nsga-ii. IEEE Transactions on Power Systems 30(2), 857866 (2015). DOI 10.1109/TPWRS.2014.2339352

33. Yue, C., Qu, B., Liang, J.: A multiobjective particle swarm optimizer using ring topology for solving multimodal multiobjective problems. IEEE Transactions on Evolutionary Computation 22(5), 805-817 (2018). DOI 10.1109/TEVC.2017.2754271

34. Zhabitskaya, E., Zhabitsky, M.: Asynchronous differential evolution with restart. In: I. Dimov, I. Faragó, L. Vulkov (eds.) Numerical Analysis and Its Applications, Lecture Notes in Computer Science, vol. 8236, pp. 555-561. Springer Berlin Heidelberg (2013). DOI 10. 1007/978-3-642-41515-9_64. URL http://dx.doi.org/ 10.1007/978-3-642-41515-9_64

35. Zhou, A., Zhang, Q., Jin, Y.: Approximating the set of pareto-optimal solutions in both the decision and objective spaces by an estimation of distribution algorithm. IEEE Transactions on Evolutionary Computation 13(5), 1167-1189 (2009). DOI 10.1109/TEVC.2009.2021467

36. Zăvoianu, A.C., Lughofer, E., Koppelstätter, W., Weidenholzer, G., Amrhein, W., Klement, E.P.: Performance comparison of generational and steady-state asynchronous multi-objective evolutionary algorithms for computationally-intensive problems. KnowledgeBased Systems 87(C), 47-60 (2015). DOI 10.1016/j. knosys.2015.05.029. URL http://dx.doi.org/10.1016/ j.knosys.2015.05.029 